\title{
In-Flight Aeroelastic Stability of the Thermal Protection System on the NASA HIAD, Part I: Linear Theory
}

\author{
Benjamin D. Goldman* and Earl H. Dowell ${ }^{\dagger}$ \\ Duke University, Durham, NC, 27708, USA \\ Robert C. Scott ${ }^{\ddagger}$ \\ NASA Langley Research Center, Hampton, VA, 23681, USA
}

\begin{abstract}
Conical shell theory and piston theory aerodynamics are used to study the aeroelastic stability of the thermal protection system (TPS) on the NASA Hypersonic Inflatable Aerodynamic Decelerator (HIAD). Structural models of the TPS consist of single or multiple orthotropic conical shell systems resting on several circumferential linear elastic supports. The shells in each model may have pinned (simply-supported) or elastically-supported edges. The Lagrangian is formulated in terms of the generalized coordinates for all displacements and the Rayleigh-Ritz method is used to derive the equations of motion. The natural modes of vibration and aeroelastic stability boundaries are found by calculating the eigenvalues and eigenvectors of a large coefficient matrix. When the in-flight configuration of the TPS is approximated as a single shell without elastic supports, asymmetric flutter in many circumferential waves is observed. When the elastic supports are included, the shell flutters symmetrically in zero circumferential waves. Structural damping is found to be important in this case. Aeroelastic models that consider the individual TPS layers as separate shells tend to flutter asymmetrically at high dynamic pressures relative to the single shell models. Several parameter studies also examine the effects of tension, orthotropicity, and elastic support stiffness.
\end{abstract}

\section{Nomenclature}

$a_{n}, b_{n}, c_{n} \quad$ Modal coordinates for the three shell displacements, $\mathrm{m}$

$D_{\text {eff }} \quad$ Effective material bending stiffness, $\mathrm{Pa} \mathrm{m}$

$D_{y}, D_{\theta} \quad$ Shell Bending stiffness in the $y$ and $\theta$ directions, respectively, $\mathrm{Pa} \mathrm{m}^{3}$

$D_{y \theta} \quad$ Shell in-plane twisting stiffness, $P a \mathrm{~m}^{3}$

$E \quad$ Material Young's Modulus, $P a$

$E_{y}, E_{\theta} \quad$ Shell Young's Modulus in the $y$ and $\theta$ directions, respectively, $P a$

$f \quad$ Frequency, $\mathrm{Hz}$

$f_{\text {crit }} \quad$ Critical frequency, at the flutter or divergence boundary, $\mathrm{Hz}$

$G_{y \theta} \quad$ Shear modulus, $P a$

$h \quad$ Shell thickness, $m$

$k \quad$ Circumferential wavenumber

$k_{\text {crit }} \quad$ Critical circumferential wavenumber, at the flutter or divergence boundary

$K_{s} \quad$ Spring stiffness for the circumferential elastic supports, $\mathrm{Pa}$

$K^{P Y R} \quad$ Pyrogel spring stiffness, $\mathrm{Pa} / \mathrm{m}$

$m \quad$ Shell mass per area, $\mathrm{kg} / \mathrm{m}^{2}$

\footnotetext{
${ }^{*}$ Graduate Student, Department of Mechanical Engineering and Materials Science, Box 90300 Hudson Hall, Student Member AIAA

${ }^{\dagger}$ William Holland Professor, Department of Mechanical Engineering and Materials Science, Box 90300 Hudson Hall, Honorary Fellow AIAA

${ }^{\ddagger}$ Senior Aerospace Engineer, Aeroelasticity Branch, NASA Langley Research Center, and Associate Fellow AIAA
} 


\begin{tabular}{|c|c|}
\hline$m^{P Y R}$ & Pyrogel mass per area, $\mathrm{kg} / \mathrm{m}^{2}$ \\
\hline$M$ & Mach number \\
\hline$n$ & Axial mode number \\
\hline$n_{\text {crit }}$ & Critical axial mode number, at the flutter or divergence boundary \\
\hline$N_{y, t o t}^{a}$ & Total applied in-plane force in $\mathrm{y}$-direction, $N / m$ \\
\hline$N_{y}^{a}$ & Applied tension in y-direction, $N / m$ \\
\hline$N_{\theta, t o t}^{a}$ & Total applied in-plane force in $\theta$-direction, $N / m$ \\
\hline$N_{x}^{a}, N_{y}^{a}, N_{x y}^{a}$ & In-plane forces applied to the square woven fabric sample, $\mathrm{N} / \mathrm{m}$ \\
\hline$p$ & Complex frequency in the aeroelastic problem, $\mathrm{rad} / \mathrm{s}$ \\
\hline$p_{s}$ & Static pressure differential, $P a$ \\
\hline$q$ & Dynamic pressure, $P a$ \\
\hline$q_{\text {crit }}$ & Critical dynamic pressure, at the flutter or divergence boundary, $\mathrm{Pa}$ \\
\hline$r_{1}, r_{2}$ & Shell radii at the minor and major ends, respectively, $m$ \\
\hline$u, v, w$ & Shell displacements, $m$ \\
\hline$w^{N}, w^{K}$ & Out-of-plane displacements of the Nextel and AKK shells, respectively, $m$ \\
\hline$U_{\infty}$ & Free-stream flow velocity, $\mathrm{m} / \mathrm{s}$ \\
\hline$y$ & Coordinate along the shell meridian \\
\hline$y_{1}, y_{2}$ & Locations of the shell minor and major ends, respectively \\
\hline $\bar{y}$ & Dimensionless shell coordinate, $\left(y-y_{1}\right) /\left(y_{2}-y_{1}\right)$ \\
\hline$\alpha$ & Shell half-cone angle \\
\hline$\Delta p$ & Total dynamic pressure, $P a$ \\
\hline$\epsilon_{y}, \epsilon_{\theta}, \epsilon_{y \theta}$ & Middle surface strains in the $y, \theta, y \theta$ directions, respectively \\
\hline$\zeta_{n}$ & Structural damping ratio for the $n^{t h}$ natural mode \\
\hline$\eta$ & Transformed (dimensionless) shell meridional coordinate for the Legendre polynomials \\
\hline$\theta$ & Shell circumferential coordinate \\
\hline$\lambda_{f}$ & Dimensionless flutter parameter \\
\hline$\nu_{y}, \nu_{\theta}, \nu_{y \theta}$ & Poisson's ratio in the $y, \theta, y \theta$ directions, respectively \\
\hline$\sigma$ & Total system damping \\
\hline$\omega$ & Angular frequency, $\mathrm{rad} / \mathrm{s}$ \\
\hline$\omega_{n}$ & Angular frequency of the $n^{t h}$ natural mode, $\mathrm{rad} / \mathrm{s}$ \\
\hline
\end{tabular}

\section{Introduction}

The NASA Hypersonic Inflatable Aerodynamic Decelerator (HIAD), ${ }^{1}$ shown in Fig. 1 , is an inflatable aeroshell with a diameter significantly larger than that of current rigid aeroshells. Its size is not limited by the launch vehicle shroud, allowing heavier payloads to be delivered to higher elevations of planets like Mars. The HIAD is comprised of two separate but interacting structural components: a stacked inflatable toroid substructure and a thermal protection system (TPS). A cross-section illustration of these components is shown in Fig. 2. The TPS is flexible enough to be packed into a small volume for transport and expands with the inflation of the toroid substructure in space. However, flexible structures like the TPS can experience both static and dynamic instabilities as a result of the fluid-structure interaction. To evaluate aerothermal performance of this system, experimental tests ${ }^{2,3}$ on small TPS coupons in the NASA 8' High Temperature Tunnel (HTT) were conducted. Observations of oscillatory motion and failure of these samples during testing suggested that aeroelastic effects needed additional quantification, and theoretical model development was required. Goldman, Dowell, and $\mathrm{Scott}^{4,5}$ developed several theoretical aeroelastic models for the TPS coupons, which are flat, square, plate-like structures. A rough prediction of in-flight stability can be made from these models; however, more precise predictions require structural models consistent with the in-flight TPS geometry, i.e. a conical shell.

While the aeroelastic stability of cylindrical shells has been investigated by numerous authors, less effort has been devoted to the study of conical shells. Shulman ${ }^{6}$ was the first to study flutter of a simply-supported conical shell using a Galerkin method. Dixon and Hudson ${ }^{7}$ used a similar method to analyze the flutter and buckling of a conical shell with generalized elastic edge restraints. Sabri, ${ }^{8}$ Bismarck-Nasr,${ }^{9}$ and Sunder ${ }^{10}$ used the finite element method to study similar configurations. While the finite element method is efficient and highly accurate, substantial effort is required in setting up the necessary computational framework, even for a 
simple system. The Galerkin method is a powerful tool, but may be difficult to apply to aeroelastic systems consisting of many interacting components. An aeroelastic evaluation of the in-flight TPS configuration requires consideration of both the toroid substructure and the multiple interacting TPS layers, and possibly the constraints between them. The Rayleigh-Ritz method is a convenient tool for these types of systems, since the complicated natural boundary conditions need not be satisfied. In this case, the Lagrangian is formulated in terms of the generalized coordinates for all shell middle-surface displacements, and the Lagrange equation is applied. Piston theory aerodynamics can be incorporated as a non-conservative generalized force. The equations of motion are then written as an eigenvalue problem, ideal for the calculation of both the natural modes and aeroelastic stability boundaries of the system.

The purpose of this paper is to study theoretically the aeroelastic behavior of the TPS attached to the HIAD in flight, within the framework of linear theory. Only the TPS on the $3 \mathrm{~m}$ HIAD with a $70^{\circ}$ halfcone angle is considered here. Three structural models are developed, each consisting of one or more conical shells, with pinned or elastically-supported edges. Several circumferential middle-surface elastic supports are included to account for the presence of the toroid substructure. The shells are comprised of the constituent TPS materials, which may be orthotropic. Natural modes and aeroelastic stability boundaries are calculated for a set of nominal cases. It is also necessary to include tension in the aeroelastic models, since the TPS may be tensioned significantly during re-entry. A membrane energy incorporates tension as a linear effect when added to the Lagrangian of the system. The effect of decreasing the nominal in-plane shear modulus of the shells is also considered. This is because some of the TPS materials are woven fabrics, which are nearly inextensible in tension but very flexible in shear. Other aspects studied include the reduction of nominal elastic support stiffness and some possible approaches for simplifying the more elaborate structural models.

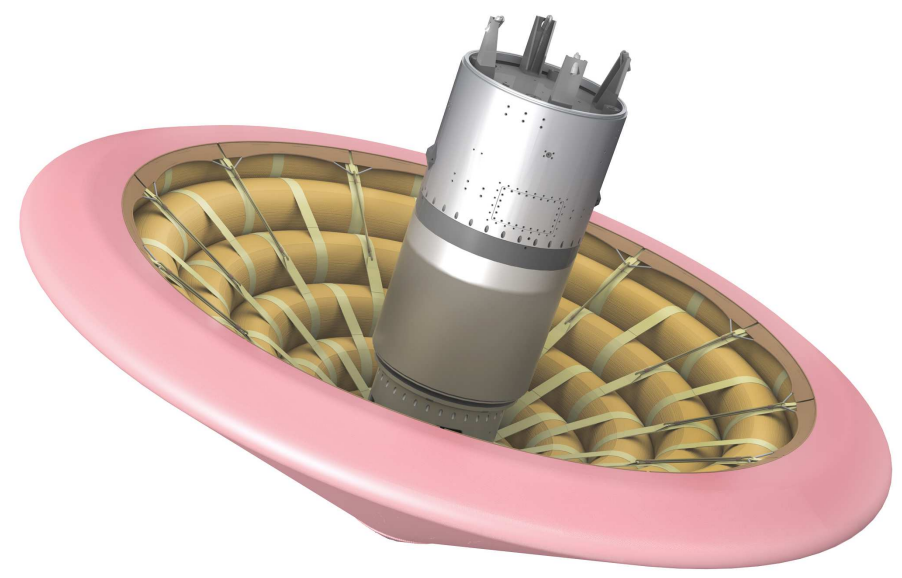

Figure 1: HIAD vehicle used in the Inflatable Re-entry Vehicle Experiment $3 .^{11}$

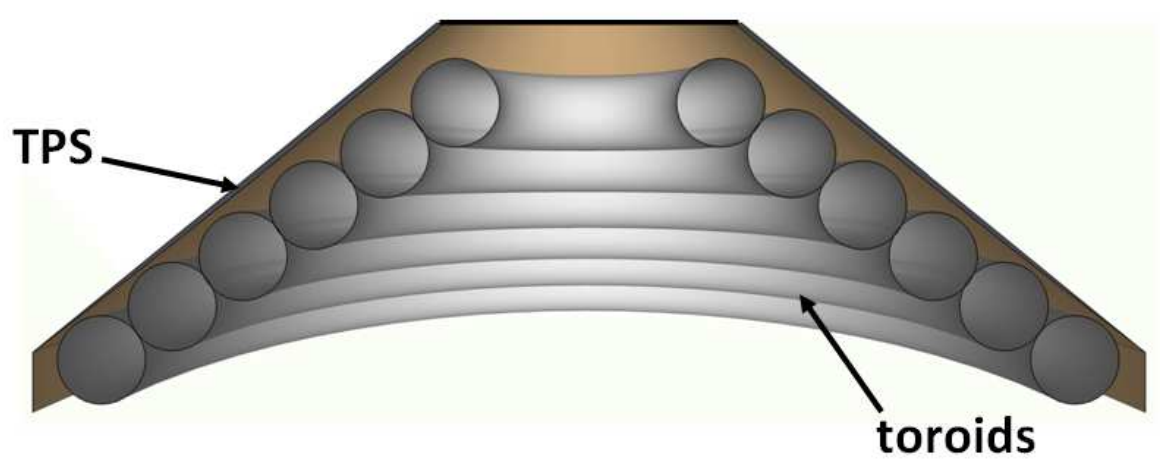

Figure 2: Approximation of the HIAD cross-section. 


\section{Components of The Thermal Protection System}

The generation 1 TPS configuration, ${ }^{3}$ illustrated in Figure 3, consists of one layer of Aluminized Kapton Kevlar (AKK), four layers of Pyrogel 2250, and two layers of Nextel 440-BF20. The AKK is comprised of Kevlar woven fabric, coated with thin layers of Aluminum and Kapton. These coatings fill the voids between the constituent fibers, such that the AKK is the most impermeable and "plate-like" TPS material. It functions as both a gas barrier and carries the structural load (tension). The Pyrogel is a soft sponge-like insulator which ensures heating of the AKK is low enough to prevent structural failure. The Nextel is a woven fabric thermal barrier that takes most of the heat load. The two Nextel layers are stitched together in various diamond patterns, along with tacks at certain point-wise locations that constrain all of the layers together. Several simplifications of this system will be employed in this analysis. The simpler shell models will assume the TPS is a single layer of AKK, with the presence of Nextel and Pyrogel included as additional mass. All structural models will ignore the presence of stitching and tacks between the TPS layers. The most elaborate structural model will consider all TPS materials; however, the two Nextel layers will be approximated as a single Nextel layer of twice the nominal mass. In addition, the four Pyrogel layers will be approximated by a single Pyrogel layer of four times both the nominal mass and thickness. A detailed discussion of the structural models is provided in section III.

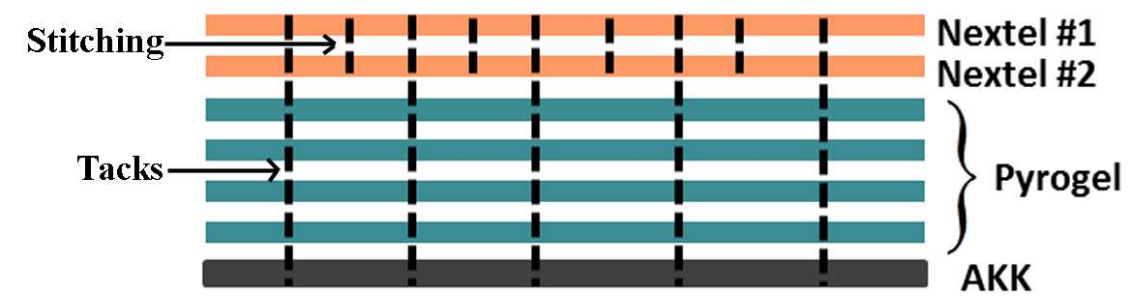

Figure 3: TPS layup consisting of $2 \times$ Nextel 440 BF-20, $4 \times$ Pyrogel 2250, and $1 \times$ AKK.

\section{II.A. TPS Material Properties}

The material properties of the individual TPS layers are provided in Table 1. The Young's moduli and Poisson ratios are obtained via material documentation, while mass, thickness, and bending stiffness were measured experimentally. The bending stiffness measurement is an overall effective stiffness based on free vibration response, so $D_{y}=D_{\theta}=D_{y \theta}=D_{\text {eff }}$ in the orthotropic shell formulation. Details of the measurement procedure can be found in Goldman, et. al. ${ }^{4}$

The Young's moduli and Poisson ratios documented here, for the Nextel and AKK, are not of the material layers themselves, but of their constituent fibers. The layers are constructed such that for a flat, rectangular sample like that shown in Fig 4, the fibers are oriented parallel to both the $\mathrm{x}$ and $\mathrm{y}$ - directions in a symmetric weave pattern. Therefore, the Young's modulus in the $\mathrm{x}$-direction is equivalent to that in the $\mathrm{y}$-direction, and can be roughly approximated by extensional Young's modulus of the fibers themselves. However, when a relatively small in-plane shear force is applied $\left(N_{x y}\right)$, the fibers rotate against each other and the sample virtually collapses (this effect is most pronounced for the Nextel, significantly less so for the AKK). This will be taken into account by using an orthotropic shell formulation, and artificially reducing the in-plane shear modulus with respect to the Young's modulus of the fibers. It will be assumed that extensional stiffness is the same in the orthogonal shell directions, $y$ and $\theta$.

\begin{tabular}{|c|c|c|c|c|c|c|}
\hline Material & $\mathrm{E}(\mathrm{Pa})$ & $\nu$ & $\mathbf{h}(\mathrm{m})$ & $\mathrm{m}\left(\mathrm{kg} / \mathrm{m}^{2}\right)$ & $\mathrm{D}_{\text {eff }}\left(\mathrm{Pa} \mathrm{m^{3 }}\right)$ & $K^{P y r},(\mathrm{~Pa} / \mathrm{m})$ \\
\hline $\begin{array}{c}\text { Nextel } \\
440-B F 20\end{array}$ & $\begin{array}{c}1.9 \mathrm{e} 11^{12} \\
\text { (fibers) }\end{array}$ & $0.26^{13}$ & $5 e-4$ & 0.46 & 0.5 & $\mathrm{~N} / \mathrm{A}$ \\
\hline AKK & $\begin{array}{c}1.24 \mathrm{e} 11^{14} \\
\text { (Kevlar } 49 \\
\text { fibers) }\end{array}$ & $0.36^{15}$ & $1.3 \mathrm{e}-4$ & 0.14 & 0.5 & $\mathrm{~N} / \mathrm{A}$ \\
\hline Pyrogel 2250 & $\begin{array}{c}3 \mathrm{e}^{16} \text { (Silica } \\
\text { Aerogel) }\end{array}$ & $0.20^{16}$ & $\begin{array}{l}8 \mathrm{e}-3(4 \\
\text { layers) }\end{array}$ & 1.36 & $\mathrm{~N} / \mathrm{A}$ & $3.9 \mathrm{e} 8$ (4 layers) \\
\hline
\end{tabular}

Table 1: Material properties of the individual TPS layers. 


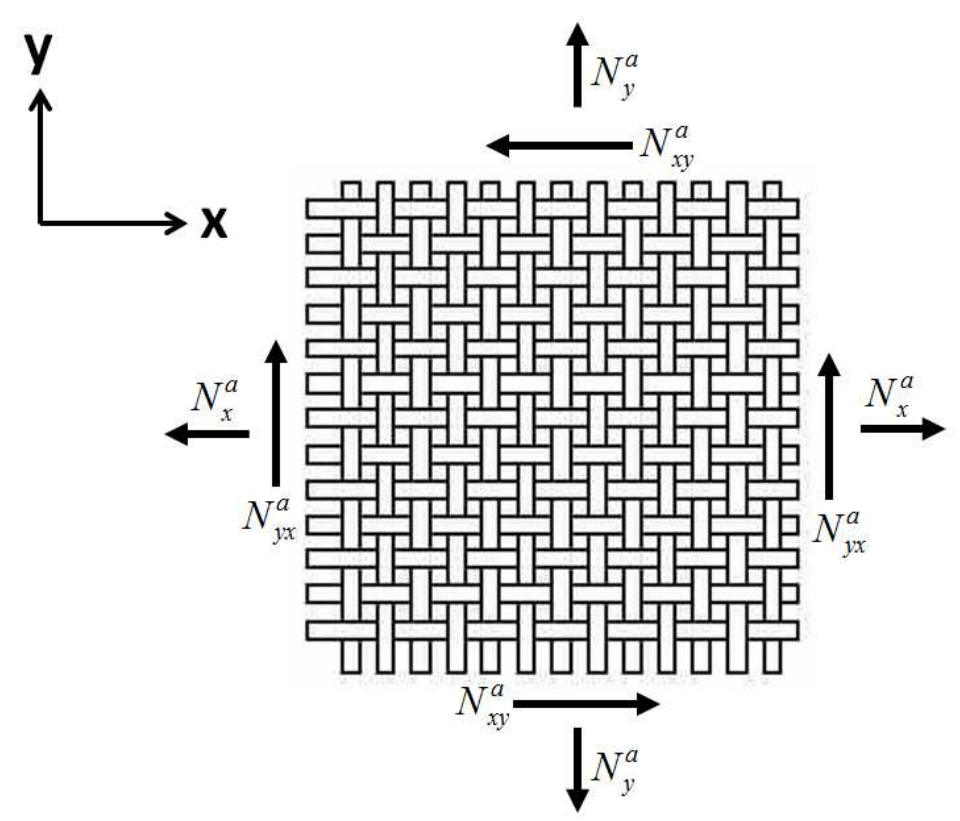

Figure 4: Flat, rectangular sample of woven fabric, similar to the TPS materials.

\section{Structural Models}

Three structural models have been developed for the TPS attached to the toroid substructure (see Figs. 1,2). Engineering illustrations of the models are given in Fig. 5. The single shell model (A) consists of a truncated conical shell with no middle surface elastic supports. It does not take into account the presence of the toroid substructure. The three separate layers of the TPS are modeled as a single layer of AKK with added mass to account for the Nextel and Pyrogel. Tension $\left(N_{y}^{a}\right)$ can also be applied at the shell boundaries. These boundaries may be pinned or free in all three displacement directions (see Fig. A1 for the shell coordinate system).

The elastically supported single shell model (B) consists of a truncated conical shell with six middle surface circumferential elastic supports. These supports take into account the presence of the toroid substructure, however, they do not consider the fact that in reality, the TPS merely rests on the surface of the substructure and is not bonded to the surfaces of the toroids. This uni-directional interaction cannot be considered using linear theory (Goldman, Dowell and $\mathrm{Scott}^{4}$ do consider this nonlinear problem for the square TPS coupons). The locations of the elastic supports are fixed at the following shell middle surface locations: $\bar{y}=0,0.2$, $0.4,0.6,0.8,1$. These are the approximate locations of the toroids on the $3 \mathrm{~m}, 70^{\circ} \mathrm{HIAD}$ substructure. The stiffness is the same for all six supports, and a parameter study will consider the aeroelastic effects of varying this stiffness. As in the previous case, the three separate layers of the TPS are modeled a single layer of AKK with added mass for the Nextel and Pyrogel, and tension can be applied. If the boundaries are pinned in all displacements, the elastic supports at the edges $(\bar{y}=0,1)$ do not affect the system. However, if the boundaries are (geometrically) free in all displacements, then the elastic supports located there limit out-of-plane deflection but do not constrain in-plane displacements.

The elastically supported three-layer shell model (C) consists of one AKK shell and one Nextel shell separated by a Pyrogel linear spring-layer. The Nextel shell is one layer of Nextel material with twice the nominal mass, as the two layers of Nextel are stitched together in the TPS layup. Since the Pyrogel is a soft, sponge-like material, it will be included as a linear spring layer. This spring-layer is assumed to be bonded to the top and bottom of the AKK and Nextel surfaces, respectively. Therefore, the presence of the Pyrogel adds stiffness and mass to the system in a manner not captured by the previous two single-layer models. The boundary conditions are the same for all layers, however, the elastic supports are only connected to the AKK surface, at the same middle surface locations in the previous model. Tension will only be applied to the AKK shell, since this is how the TPS is restrained to the HIAD. 


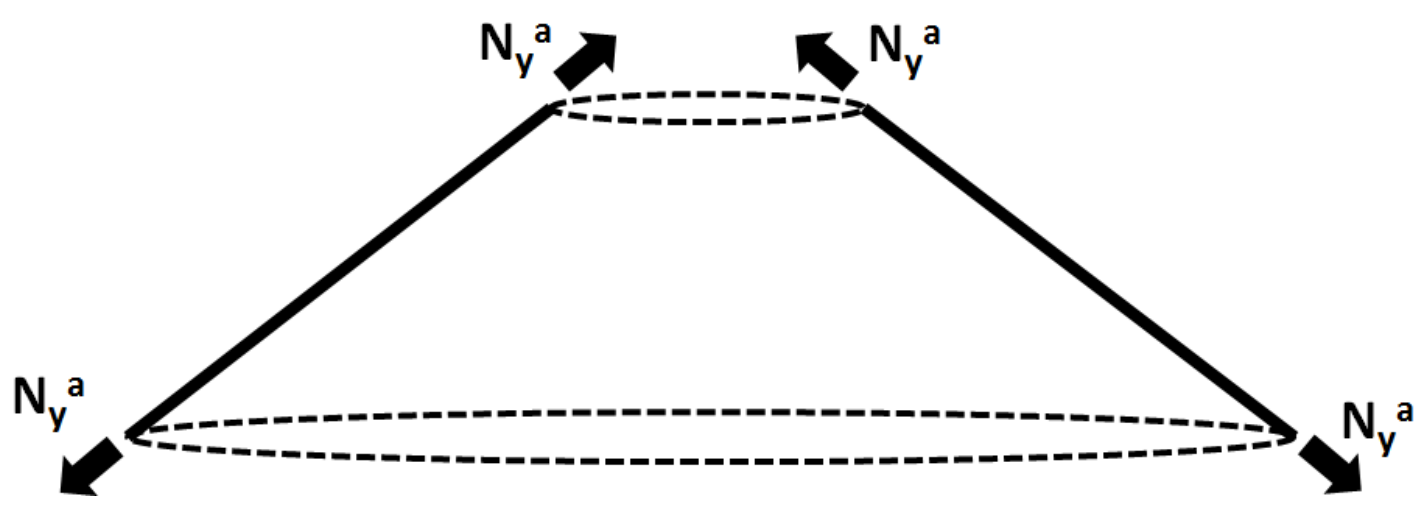

Model A: Single shell.

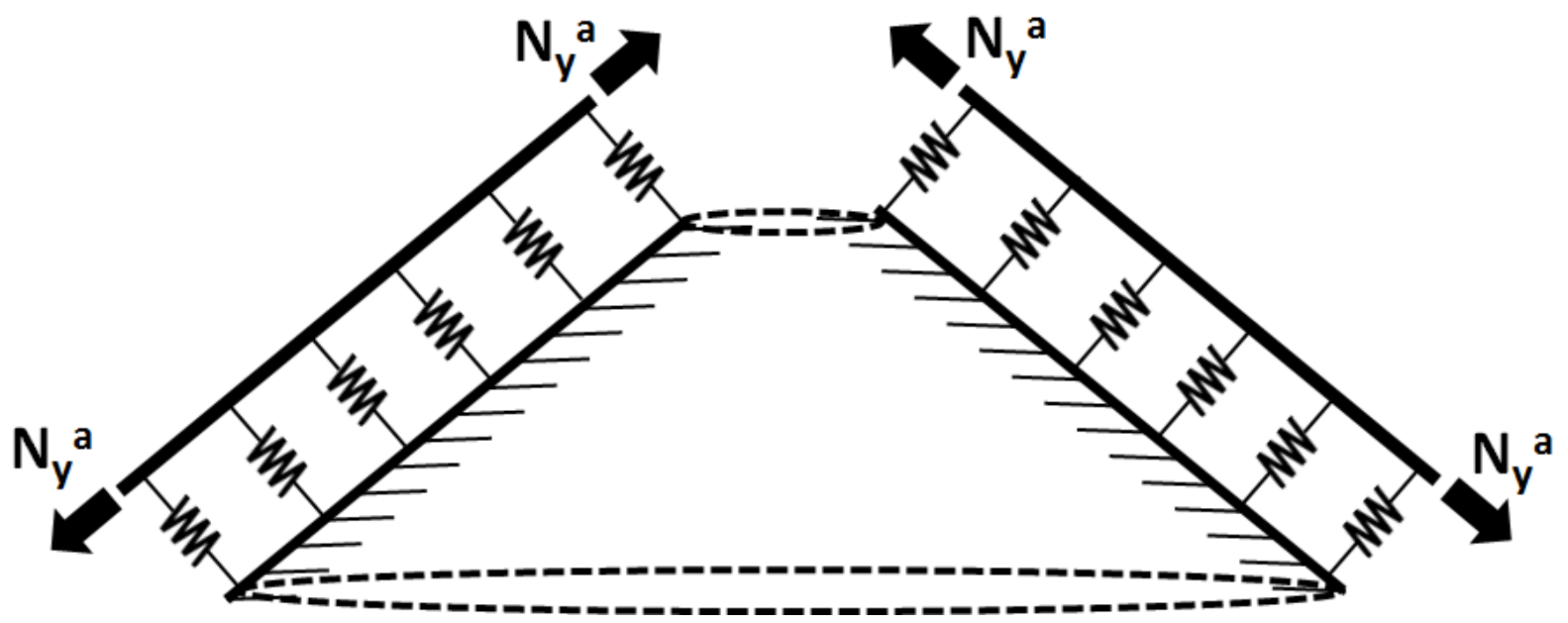

Model B: Elastically supported single shell.

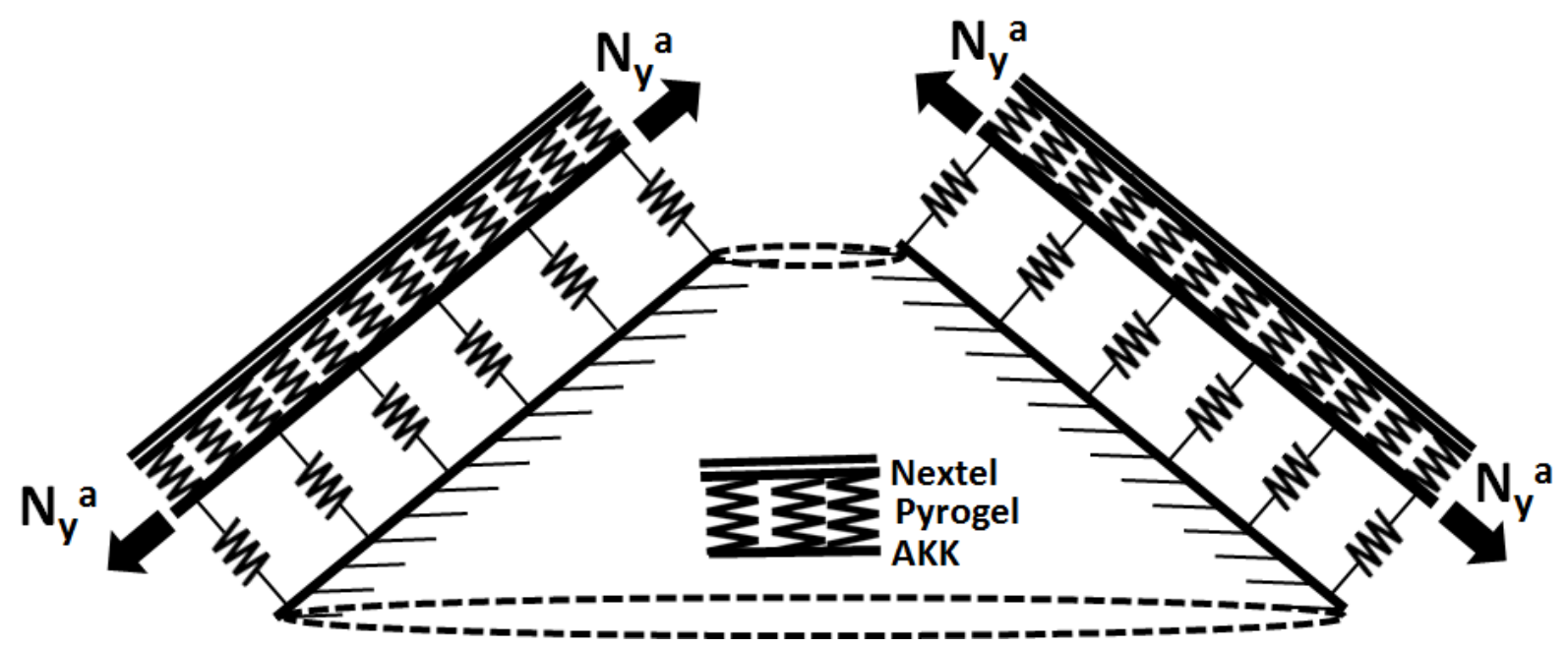

Model C: Elastically supported three-layer shell.

Figure 5: Conical shell structural models of the TPS on the HIAD. 


\section{Aeroelastic Equations of Motion and Computational Methods}

The Rayleigh-Ritz method is used to derive the aeroelastic equations of motion for all conical shell structural models subject to a local supersonic flow. The displacements in each shell are expanded using assumed mode shapes that satisfy the geometric boundary conditions only, and the Lagrangian is expressed in terms of the displacement modal coordinates. The governing equations are written in block matrix form, and solutions are found by examining the eigenvalues and eigenvectors of the system. The method allows for computation of natural frequencies and natural mode shapes, as well as the flutter and divergence boundaries, frequencies, and mode shapes.

Linearized Donnell thin shell theory ${ }^{7,17}$ is used to formulate the strain-displacement and curvaturedisplacement relations for the truncated conical shells. The effect of orthotropicity is included by using different Young's moduli, Poisson ratios, and bending stiffness in the shell coordinate directions. Piston theory with the curvature correction is used for the aerodynamic pressure.

\section{IV.A. Model A: The single shell}

The strains and curvatures are:

$$
\begin{gathered}
\varepsilon_{y}=\frac{\partial u}{\partial y} \\
\varepsilon_{\theta}=\frac{u-w \cot \alpha}{y}+\frac{1}{y \sin \alpha} \frac{\partial v}{\partial \theta} \\
\varepsilon_{y \theta}=\frac{\partial v}{\partial y}-\frac{v}{y}+\frac{1}{y \sin \alpha} \frac{\partial u}{\partial \theta} \\
\chi_{y}=\frac{\partial^{2} w}{\partial y^{2}} \\
\chi_{\theta}=\frac{1}{y} \frac{\partial w}{\partial y}+\frac{1}{y^{2} \sin ^{2} \alpha} \frac{\partial^{2} w}{\partial \theta^{2}} \\
\chi_{y \theta}=\frac{1}{y \sin \alpha} \frac{\partial^{2} w}{\partial y \partial \theta}-\frac{1}{y^{2} \sin \alpha} \frac{\partial w}{\partial \theta}
\end{gathered}
$$

The force and moment relations are:

$$
\begin{aligned}
& N_{y}=\frac{E_{y} h}{1-\nu_{y} \nu_{\theta}}\left(\varepsilon_{y}+\nu_{\theta} \varepsilon_{\theta}\right) \\
& N_{\theta}=\frac{E_{\theta} h}{1-\nu_{\theta} \nu_{y}}\left(\varepsilon_{\theta}+\nu_{y} \varepsilon_{y}\right) \\
& N_{y \theta}=G_{y \theta} h \varepsilon_{y \theta} \\
& M_{y}=-D_{y}\left(\chi_{y}+\nu_{\theta} \chi_{\theta}\right) \\
& M_{\theta}=-D_{\theta}\left(\chi_{\theta}+\nu_{y} \chi_{y}\right) \\
& M_{y \theta}=-D_{y \theta} \chi_{y \theta}
\end{aligned}
$$

The shell strain energy is composed by integrating over the entire shell the forces $\times$ strains and moments $\times$ curvatures:

$$
U_{S}=\frac{1}{2} \int_{y_{1}}^{y_{2}} \int_{0}^{2 \pi}\left(N_{y} \varepsilon_{y}+N_{\theta} \varepsilon_{\theta}+N_{y \theta} \varepsilon_{y \theta}-M_{y} \chi_{y}-M_{\theta} \chi_{\theta}-2 M_{y \theta} \chi_{y \theta}\right) y \sin \alpha d \theta d y
$$

Applied in-plane loading (tension) and a static pressure differential can be taken into account with linear theory by assuming that these forces result in only infinitesimal strains, and a state of initial stress. The membrane energy associated with this stress is given by McNeal: ${ }^{18}$

$$
U_{I S}=\frac{1}{2} \int_{y_{1}}^{y_{2}} \int_{0}^{2 \pi}\left(N_{y, t o t}^{a} \Theta_{\theta}^{2}+N_{\theta, t o t}^{a} \Theta_{y}^{2}+\left\{N_{y, t o t}^{a}+N_{\theta, t o t}^{a}\right\} \Theta_{n}^{2}\right) y \sin \alpha d \theta d y
$$

where the strain rotation vectors are:

$$
\begin{aligned}
& \Theta_{\theta}=-\frac{\partial w}{\partial y} \\
& \Theta_{y}=\frac{1}{y \sin \alpha}\left\{v-\frac{\partial w}{\partial \theta}\right\} \\
& \Theta_{n}=-\frac{1}{2 y \sin \alpha} \frac{\partial u}{\partial \theta}+\frac{1}{2} \frac{\partial v}{\partial x}
\end{aligned}
$$

The total applied in-plane forces are related to the static pressure differential and tension by the relation:

$$
\begin{aligned}
& N_{y, t o t}^{a}=-\frac{y \tan \alpha p_{s}}{2}-N_{y}^{a} \\
& N_{\theta, t o t}^{a}=-y \tan \alpha p_{s}
\end{aligned}
$$


where $p_{s}$ is the static pressure differential across the shell, and $N_{y}^{a}$ is the tension applied at the shell edges, acting in along the shell meridian (y-direction) only.

The kinetic energy, neglecting rotatory inertia, is:

$$
T=\frac{m}{2} \int_{y_{1}}^{y_{2}} \int_{0}^{2 \pi}\left[\left(\frac{\partial u}{\partial t}\right)^{2}+\left(\frac{\partial v}{\partial t}\right)^{2}+\left(\frac{\partial w}{\partial t}\right)^{2}\right] y \sin \alpha d \theta d y
$$

The virtual work is:

$$
\delta W=-\int_{y_{1}}^{y_{2}} \int_{0}^{2 \pi} \Delta p \delta w y \sin \alpha d \theta d y
$$

Piston theory is used for the aerodynamic pressure. The curvature correction term for cylindrical shells, given by Krumhaar ${ }^{19}$ is also included, and modified here for conical shells:

$$
\Delta p=\frac{2 q}{M}\left[\frac{\partial w}{\partial y}+\frac{1}{U_{\infty}} \frac{\partial w}{\partial t}-\frac{1}{2 M} \frac{w}{y \sin \alpha}\right]
$$

Next, the three shell displacements expanded in modal form:

$$
\begin{aligned}
& u(y, \theta, t)=\sum_{n} \hat{a}_{n} e^{p t} \cos k \theta \phi_{n}(y) \\
& v(y, \theta, t)=\sum_{n} \hat{b}_{n} e^{p t} \sin k \theta \phi_{n}(y) \\
& w(y, \theta, t)=\sum_{n} \hat{c}_{n} e^{p t} \cos k \theta \phi_{n}(y)
\end{aligned}
$$

The modes in the circumferential direction are sinusoidal functions of the circumferential wavenumber $k$, satisfying the periodicity condition around the shell. They also allow for asymmetric displacements about the shell axis. The modal functions in the axial direction must satisfy the geometric boundary conditions at both ends of the shell. On the HIAD vehicle, no constraints on deflection exist at the major end of the TPS shell, but the largest toroid provides circumferential elastic support. The minor end edge is secured in a similar fashion, elastically supported by the smallest toroid. Though not physically representative of the HIAD, as a first approximation we consider modal functions that restrict all shell displacements at the boundaries:

$$
\left.u\right|_{y=y_{1}, y_{2}}=\left.v\right|_{y=y_{1}, y_{2}}=\left.w\right|_{y=y_{1}, y_{2}}=0
$$

For conical shells, these modal functions are:

$$
\phi_{n}(y)=\sin \left(n \pi\left\{\frac{y-y_{1}}{y_{2}-y_{1}}\right\}\right)
$$

Another approach is to use modes that do not satisfy any constraints on displacements at the boundaries. In this case, ordinary polynomials are typically chosen, and have been used in previous investigations of cylindrical and conical shell free-vibrations. However, this results in ill-conditioned coefficient matrices when more than five or six modes are used. Since an aeroelastic evaluation may require a substantial number of modes for a converged solution, we instead use Legendre polynomials. The orthogonality of these functions results in a numerically robust formulation. The following recursive definition is used to generate the polynomials in a discrete numerical procedure:

$$
(n+1) P_{n+1}(\eta)=(2 n+1) P_{n}(\eta) \eta-n P_{n-1}(\eta)
$$

Since Legendre polynomials are defined on the interval $[-1,1]$, the shell coordinate $y$ is transformed to a suitable coordinate $\eta$ :

The Lagrangian of the system is:

$$
\eta=\frac{2 y-\left(y_{2}+y_{1}\right)}{y_{2}-y_{1}}
$$

$$
L=T-\left\{U_{S}+U_{I S}\right\}
$$

The equations of motion are formulated by substituting Eq. 12 into Eq. 17, and applying the Lagrange equation for each displacement modal coordinate. At this point, the following expression for modal structural damping can be included in the equations for $\hat{c}_{n}$.

$$
Z_{n j}=2 \zeta_{n} \omega_{n} M_{n j}
$$


After expressing the equations in matrix form, the eigenvalue problem for aeroelastic stability is:

$$
\left[p^{2} \boldsymbol{M}+p \boldsymbol{C}+\boldsymbol{K}\right]\left\{\begin{array}{l}
\hat{a} \\
\hat{b} \\
\hat{c}
\end{array}\right\}=0
$$

where $\boldsymbol{M}, \boldsymbol{C}$, and $\boldsymbol{K}$ are the mass, damping, and stiffness matrices defined by:

$$
\boldsymbol{M}=\left[\begin{array}{ccc}
G & 0 & 0 \\
0 & H & 0 \\
0 & 0 & M
\end{array}\right] \quad \boldsymbol{C}=\left[\begin{array}{ccc}
0 & 0 & 0 \\
0 & 0 & 0 \\
0 & 0 & Z_{A}+Z_{S D}
\end{array}\right] \quad \boldsymbol{K}=\left[\begin{array}{ccc}
A+J & B+K & D \\
B^{T}+K^{T} & C+L & E+N \\
D^{T} & E^{T}+N^{T} & F+R+Q
\end{array}\right]
$$

The coefficient submatrices in Eq. 20 are provided in the appendix.

To calculate the natural frequencies and mode shapes of the shell, a value for the circumferential wavenumber is selected, and the aerodynamic submatrices $Q$ and $Z_{A}$ are set to zero. The eigenvalue problem in Eq. 19 is then solved using a numerical mathematics toolbox. The resulting eigenvalues are the natural frequencies for the shell axial modes, at the selected circumferential wavenumber. The eigenvectors are sets of coefficients which can be used along with the modal expansions in Eq. 12 to construct the natural mode shapes.

To analyze aeroelastic stability, the aerodynamic submatrices $Q$ and $Z_{A}$ are included, and the eigenvalue problem is solved for a given circumferential wavenumber and dynamic pressure. Unlike the natural frequency calculation, however, the eigenvalues are now complex numbers of the form:

$$
p=\sigma+i \omega
$$

where $\sigma$ is the total system damping and $\omega$ is the frequency. The system is unstable when $\sigma=0$ for any eigenvalue, which will occur when the aerodynamic pressure reaches a critical value. The value of $\omega$ corresponding to $\sigma=0$ can be zero, in the case of divergence, or nonzero, in the case of flutter. In supersonic flow, flutter is usually characterized by the coalescence of two natural structural modes, resulting in a single flutter frequency.

\section{IV.B. Model B: Elastically supported single shell}

The elastic supports are included using the following potential energy expression:

$$
U_{E S}=\left.\frac{K_{s}}{2} \int_{0}^{2 \pi} \sum_{i}\left(w^{2} y\right)\right|_{y=y_{i}} \sin \alpha d \theta
$$

where index $i$ refers to a given support. The inertia of the supports is neglected since the toroid substructure on the HIAD is large, massive, and relatively stiff. The shell is assumed to be continuously bonded to the supports along the circumferential direction.

The Lagrangian is now:

$$
L=T-\left\{U_{S}+U_{I S}+U_{E S}\right\}
$$

Applying the Lagrange equation results in an eigenvalue problem similar to Eq. 19 with submatrix $S$ (provided in the Appendix) added to the submatrix $F$ in the stiffness matrix $\boldsymbol{K}$.

\section{IV.C. Model C: The elastically supported three-layer shell}

The three layer shell is comprised of the Nextel and AKK shells, separated by a Pyrogel spring-layer. The constitutive laws, strain-displacement relations, and total strain energy for each shell in this model are the same as the single shell in section IV.A. The elastic supports are also included in the same manner as before.

The potential and kinetic energies of the Pyrogel are:

$$
U^{P Y R}=\frac{K^{P Y R}}{2} \int_{y_{1}}^{y_{2}} \int_{0}^{2 \pi}\left(w^{N}-w^{K}\right)^{2} y \sin \alpha d \theta d y
$$




$$
T^{P Y R}=\frac{m^{P Y R}}{2} \int_{y_{1}}^{y_{2}} \int_{0}^{2 \pi}\left(\frac{\partial w^{N}}{\partial t}+\frac{\partial w^{K}}{\partial t}\right)^{2} y \sin \alpha d \theta d y
$$

The Lagrangian is now:

$$
L=T^{N}+T^{K}+T^{P Y R}-\left\{U_{S}^{N}+U_{S}^{K}+U_{I S}^{N}+U_{I S}^{K}+U_{E S}^{K}+U^{P Y R}\right\}
$$

where superscripts $N$ and $K$ refer to the Nextel and AKK, respectively. Note that the potential energy of the circumferential elastic supports in this case is a function of the AKK deflection only, since there is no direct contact between the Nextel and the supports in the structural model. In addition, the aerodynamic generalized forces are only included in the equations for the Nextel shell, since it is the only structural component exposed directly to flow.

The three displacements in each shell are expanded using the modal series in Eq. 12, for a total of six modal coordinates. It is assumed that the Nextel and AKK have the same displacement boundary conditions, such that the same axial modal functions can be used for each shell. However, the Nextel and AKK as individual shells may experience flutter or divergence for a given dynamic pressure at different critical circumferential wavenumbers. To determine if this is still the case for the two shells in the elastically supported three-layer shell model, we consider the following Pyrogel coupling term (for both inertial and stiffness coupling):

$$
\sum_{n} \sum_{i} \int_{0}^{2 \pi} \cos \left(k^{N} \theta\right) \cos \left(k^{K} \theta\right) d \theta \int_{y 1}^{y 2} \phi_{n} \phi_{i} y \sin \alpha d y
$$

Orthogonality of the circumferential modes indicates that no coupling between the two shells exists when $k^{N} \neq k^{K}$. Since the Nextel cannot deflect without inducing deflection in the AKK, the two shells in this model must undergo flutter or divergence in the same number of circumferential waves.

The eigenvalue problem has the same form as Eq. 19, and the mass, damping, and stiffness matrices are now given by:

$$
\begin{aligned}
& \mathbf{M}=\left[\begin{array}{cccccc}
G^{N} & 0 & 0 & 0 & 0 & 0 \\
0 & H^{N} & 0 & 0 & 0 & 0 \\
0 & 0 & M^{N}+V^{P Y R} & 0 & 0 & V^{P Y R} \\
0 & 0 & 0 & G^{K} & 0 & 0 \\
0 & 0 & 0 & 0 & H^{K} & 0 \\
0 & 0 & V^{P Y R} & 0 & 0 & M^{K}+V^{P Y R}
\end{array}\right] \\
& \mathbf{C}=\left[\begin{array}{cccccc}
0 & 0 & 0 & \ddots & & \\
0 & 0 & 0 & & 0 & \\
0 & 0 & Z_{S D}^{N}+Z_{A}^{N} & & & \ddots \\
\ddots & & & & 0 & 0 \\
& 0 & & 0 & 0 & 0 \\
& & \ddots & 0 & 0 & Z_{S D}^{K}+Z_{A}^{K}
\end{array}\right]
\end{aligned}
$$

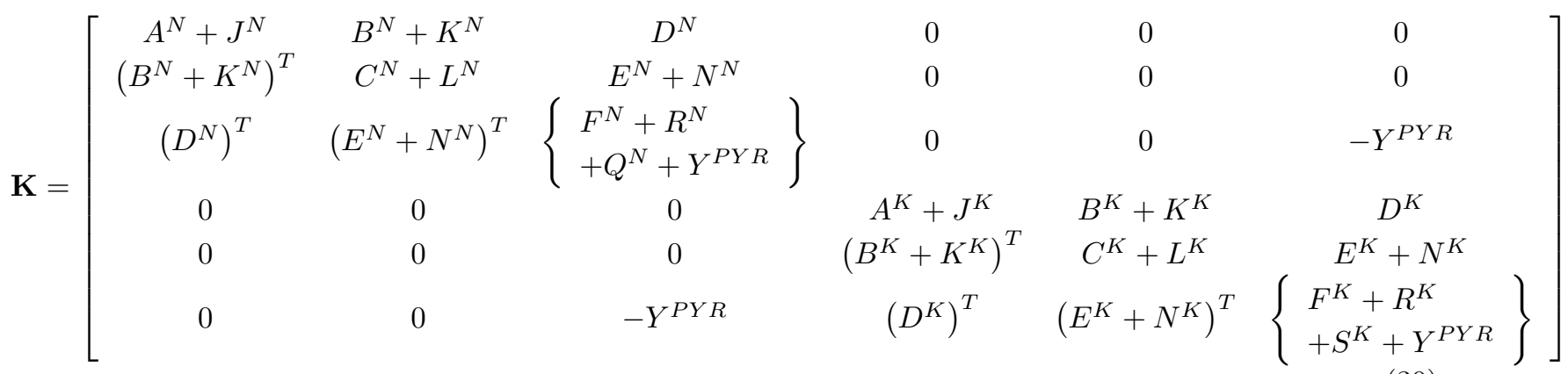




\section{Results}

Prior to discussing the TPS models, the first set of results in this section will compare the natural modes and aeroelastic stability boundaries for single, isotropic, small angle conical shells to previously published theoretical and experimental data. These shells are not constructed of TPS materials and are only discussed here for model validation purposes. The first results section pertaining to the TPS consists of a series of tables containing the nominal test cases and system parameters used throughout this paper. Natural frequencies and mode shapes are calculated for these nominal cases, and the effect of large tension on the behavior of the frequencies is also examined. Aeroelastic solutions are also computed for these cases, and the effect of tension, shear modulus, and circumferential support stiffness will also be considered in a series of parameter studies.

\section{V.A. Model validation}

While no previously published studies of multiple layer conical shells on circumferential elastic supports currently exist, there is an abundance of earlier work on the free vibrations and flutter of a single conical shell with either pinned or free edges. Recall that the TPS single-shell model (A) is exactly this configuration, so some degree of validation can be accomplished through comparison to earlier work, even if the geometric and material properties are different. We first consider the $45^{\circ}$ steel conical shell with free edges in $\mathrm{Hu}$, Gormley, and Lindholm. ${ }^{20}$ They measured experimentally the natural frequencies and mode shapes as a function of circumferential wavenumber. A comparison between the present theory and their experiment is given in Fig. 6. The dimensionless frequency parameter used in the comparison is defined as:

$$
\Omega=\omega r_{1} \sqrt{\frac{m\left(1-\nu_{y}^{2}\right)}{h E_{y}}}
$$

There is generally good agreement for the first two axial modes, while the theoretical result is slightly higher for the third mode. Experimental natural frequencies were unavailable for higher axial modes. A comparison of the theoretical and experimental mode shapes is given in Fig. 7. There is also good agreement between theory and experiment for the first and second modes. It is observed that at low values of circumferential wavenumber $(k=4,6)$, the mode shapes are rigid-body-like, though the corresponding natural frequencies are nonzero. The dynamics of a free-free shell will be revisited in the discussion of TPS model A-2 in section V.D.1.

A comparison of flutter boundaries for a $5^{\circ}$ conical shell with pinned (simply-supported) edges is given in Table 2. The earlier works present the flutter dynamic pressure as a dimensionless parameter defined by:

$$
\lambda_{f}=\frac{2 q_{f} r_{1}^{2}}{D_{y} \sqrt{M^{2}-1}}
$$

For comparison purposes, the flutter boundary for the current work is also given in terms of this quantity, but will be presented in dimensional form throughout the rest of the paper. The flutter boundary for this shell configuration is calculated using two methods. First, the sinusoidal modal functions in Eq. 14 are used, resulting in a flutter boundary higher than that of Sabri and Dixon but lower than that of Shulman. Second, Lengendre polynomials and spring supports with large $K_{s}$ in all three displacement directions are used to replicate the pinned condition in all displacement directions. This is to verify that Legendre polynomials are also sufficient modal functions for the aeroelastic stability problem. The flutter boundary for this case is only slightly lower than the previous one with sinusoidal modal functions. Both computations have the same critical flutter circumferential wavenumber of 6, which is consistent with Shulman and Sabri. Good agreement between the current and earlier works for a single shell, using both types of modal functions, indicates that the methods developed in this paper are valid. 


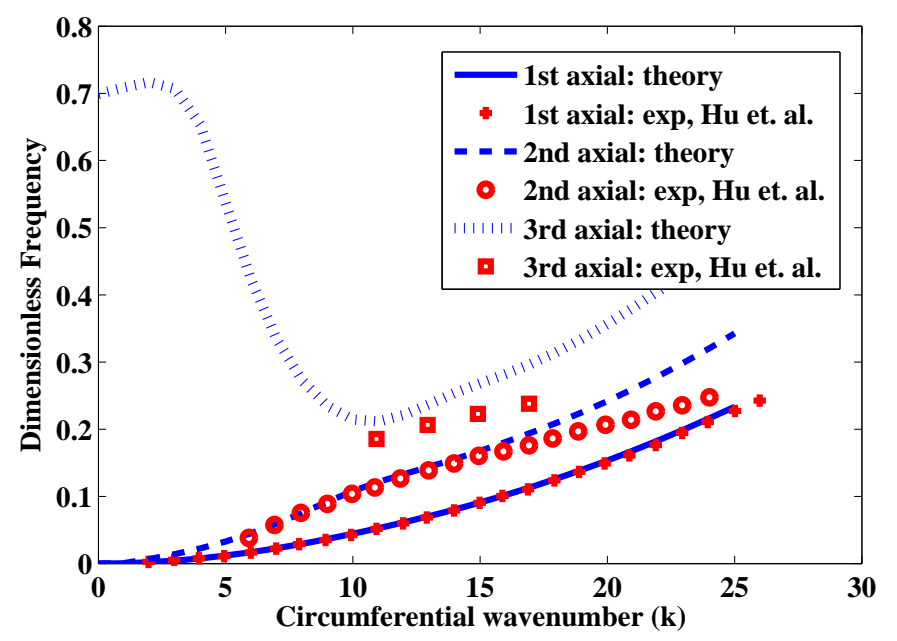

Figure 6: Theoretical and experimental natural frequencies of a $45^{\circ}$ deg conical shell with free edges, as a function circumferential wavenumber.

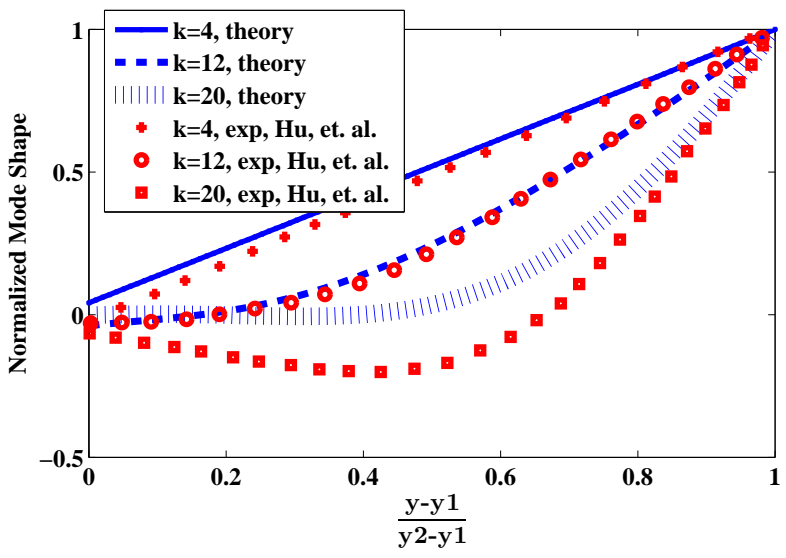

(a) 1st axial.

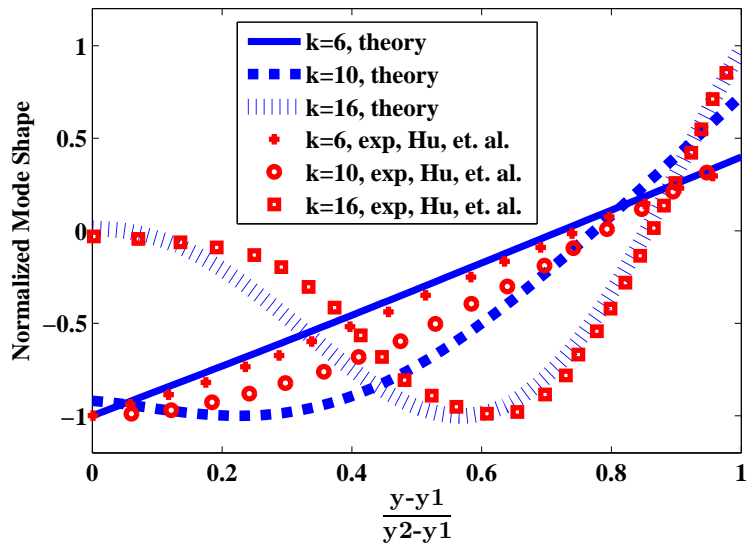

(b) 2nd axial.

Figure 7: Theoretical and experimental mode shapes as a function of circumferential wavenumber for a $45^{\circ}$ conical shell with free edges.

\begin{tabular}{clcc}
\hline Investigator & \multicolumn{1}{c}{ Method } & $\lambda_{\boldsymbol{f}}$ & $\boldsymbol{k}_{\boldsymbol{f}}$ \\
\hline Shulman $^{6}$ & $\begin{array}{l}\text { Stress function formulation, Galerkin method with assumed de- } \\
\text { flection modes satisfying geometric BCs only, 4 terms }\end{array}$ & 670 & 6 \\
\hline Dixon and Hudson $^{7}$ & $\begin{array}{l}\text { Stress function formulation, Galerkin method with assumed de- } \\
\text { flection modes satisfying geometric BCs only, 12 terms }\end{array}$ & 590 & 5 \\
\hline Sabri $^{8}$ & Hybrid finite elment method, 15 elements & 598 & 6 \\
\hline Current work & $\begin{array}{l}\text { Displacement formulation, Rayleigh-Ritz method with assumed } \\
\text { modes satisfying geometric BCs only, 12 terms }\end{array}$ & 638 & 6 \\
\hline Current work & $\begin{array}{l}\text { Displacement formulation, Rayleigh Ritz method with Legendre } \\
\text { polynomials and elastic edge restraints, 14 terms }\end{array}$ & 610 & 6 \\
\hline \hline
\end{tabular}

Table 2: Comparison of flutter boundaries for a $5^{\circ}$ conical shell with pinned edges. 


\section{V.B. Structural/aerodynamic parameters and nominal test cases}

The shell geometry will remain fixed for most calculations in this paper and is given in Table 3 (A different geometry will only be discussed in section V.D.3). These dimensions correspond approximately to that of the TPS on a $3 \mathrm{~m}$ HIAD vehicle. The Mach number, $M$, is also fixed to 2 for all calculations. Higher Mach numbers are not normally seen behind detached shocks in front of bluff bodies like the HIAD. Lower Mach numbers may be considered in a future paper, but a more elaborate aerodynamic theory would be required.

The nominal test cases are given in Table 4. In these cases, the shells are assumed to be isotropic, and $\zeta_{n}$ and $p_{s}$ are initially set to zero. Structural damping is not normally important for shell flutter??, but a later analysis may indicate that it is necessary in some of the nominal cases. Including a static pressure in the aeroelastic models has little influence on the flutter boundaries, but results in divergence near the critical buckling pressures. These critical static pressures are quite low, of order $100 \mathrm{~Pa}$ in most cases. This is because these pressures are incorporated via a membrane strain energy (Eq. 6), valid only under the assumption of infinitesimal strains. Therefore, to assess more accurately the effect of the static pressure, a nonlinear structural theory is required. Pressure-induced large deformations in the regions between the circumferential elastic supports may add stabilizing nonlinear tension to the system.

\begin{tabular}{cc}
\hline $\boldsymbol{\alpha}$ & $70^{\circ}$ \\
$\boldsymbol{r}_{1}$ & $0.25 \mathrm{~m}$ \\
$\boldsymbol{r}_{2}$ & $1.5 \mathrm{~m}$ \\
$\boldsymbol{y}_{1}$ & $0.27 \mathrm{~m}$ \\
$\boldsymbol{y}_{2}$ & $1.6 \mathrm{~m}$ \\
\hline \hline$M$ & 2 \\
\hline
\end{tabular}

Table 3: Nominal shell geometry and aerodynamic parameters.

\begin{tabular}{ccccc}
\hline Model & $\begin{array}{l}\text { Modal } \\
\text { function }\end{array}$ & $\begin{array}{l}\text { Effective boundary } \\
\text { conditions }\end{array}$ & $\boldsymbol{K}_{\boldsymbol{s}}(\boldsymbol{N} / \boldsymbol{m})$ & $\begin{array}{c}\text { Support } \\
\text { locations }(\bar{y})\end{array}$ \\
\hline A-1 & Sine function & Pinned in $u, v, w$ & 0 & N $/ \mathrm{A}$ \\
A-2 & Legendre polynomials & Free in in $u, v, w$ & 0 & N $/ \mathrm{A}$ \\
B-1 & Sine function & Pinned in $u, v, w$ & $1.00 \mathrm{E}+08$ & $0^{\dagger}, 0.2,0.4,0.6,0.8,1^{\dagger}$ \\
B-2 & Legendre polynomials & Elastically-supported in $w$ & $1.00 \mathrm{E}+08$ & $0,0.2,0.4,0.6,0.8,1$ \\
C-1 & Sine function & Pinned in $u, v, w$ & $1.00 \mathrm{E}+08$ & $0^{\dagger}, 0.2,0.4,0.6,0.8,1^{\dagger}$ \\
C-2 & Legendre polynomials & Elastically-supported in $w$ & $1.00 \mathrm{E}+08$ & $0,0.2,0.4,0.60 .8,1$ \\
\hline
\end{tabular}

Table 4: Structural parameters for the nominal test cases.

$\dagger$ In the cases with pinned BCs, elastic supports at the two shell edges have no effect, since all displacements at these locations must vanish.

\section{V.C. Natural Modes}

Before analyzing the aeroelastic behavior of these systems, the natural modes of vibration will be studied. Since the natural modes are a function of the circumferential wavenumber, $k$, aeroelastic solutions must be calculated for a wide range of $k$ values, in order to determine the critical $k$ with the lowest flutter or divergence dynamic pressure. However, an analysis of the natural modes should suggest which modes are susceptible to flutter and divergence, allowing for more efficient aeroelastic computations. In the first part of this section, the natural frequencies as a function of $k$ will be presented for all nominal cases in Table 4. Additionally, the effect of applying $1000 \mathrm{~N} / \mathrm{m}$ tension at the shell edges, for each nominal case, will be examined. For conciseness, the first three axial modes are provided for all models, though higher modes with noteworthy behavior are included where necessary. Regions of interest will be identified by red boxes in the figures. Modes contained within these regions are either likely to flutter, or may be significantly impacted by applied tension. A selection of natural mode shapes for all nominal cases, as a function of $k$, is also provided. 


\section{V.C.1. Natural Frequencies as a Function of $k$}

The natural frequencies of the first four axial modes for model A-1 are given in Fig. 8. Here, the region of interest is from $k=0$ to 3 where the frequencies of these modes are closest in separation. It is in this range of $k$ where coalescence flutter is most likely to occur. Applying $1000 \mathrm{~N} / \mathrm{m}$ tension not only increases the magnitude of the frequencies, but also increases the separation between them. This suggests that that the flutter dynamic pressure will likely increase with tension for this particular model.

The natural frequencies of the first three axial modes for model A-2 are given Fig. 9. The effect of free edges is most clearly demonstrated by examining the first two axial modes, which approach zero frequency at low $k$. This is expected, since the lack of edge restraint allows non-oscillatory rigid-body translational and rotational modes (in the axial direction only). Applying the same amount of tension as before does increase the natural frequencies and the separation between them, however, the first axial mode at $k=0$ remains at a zero frequency. This suggests that tension will have little effect on the aeroelastic behavior of this model. The zero frequency modes also indicate that divergence, rather than flutter may be more likely to occur in this case.

When the nominal circumferential elastic supports are included, in the case of model B-1, the behavior of the natural frequencies is more complicated. Figure 10 shows that there are several locations, including $k=3,10,11,16$, and 25 , where differential axial modes approach the same frequency. Upon closer inspection of the numerical values, these frequency separations may be as small as $0.2 \mathrm{~Hz}$ depending on the value of $k$. The regions of particular interest here are for $\mathrm{n}=1,2,3,6$, and 7 between $k=0$ and 3 . Without any tension, the $2^{\text {nd }}$ and $3^{\text {rd }}$ axial modes are extremely close at $k=3$, yet they separate significantly at this location when tension is applied. However, the opposite is true for the $6^{t h}$ and $7^{t h}$ axial modes. In the untensioned case, these modes are far apart at low $k$, but nearly collapse onto each other in the tensioned case. It is therefore likely that the $6^{\text {th }}$ and $7^{\text {th }}$ axial modes are more susceptible to flutter when high tension is applied to this model.

The effect of the elastically supported edges on the natural modes for the single-shell (model B-2) is demonstrated in Figure 11. There still exists a zero frequency for the first axial mode at $k=0$, however, unlike model A-2, there is a rapid increase in frequency from $k=1$ to 2. Higher axial modes are close in proximity through a range of $k$, which suggests higher mode flutter is likely in this case. There are two regions of interest here, including $k=0$ to 5 for $\mathrm{n}=2,3,4$ and $k=0$ to 5 for $\mathrm{n}=6$ and 7 . When $1000 \mathrm{~N} / \mathrm{m}$ tension is applied, the modes in the latter region not only shift to higher frequencies, but also seem to reduce in separation. It is likely that the modes in this range of $n$ and $k$ will be susceptible to flutter.

The natural frequencies of the first three axial modes for model C-1 are given in Figure 12. The frequencies at low $k$ are significantly higher than in the previous models with pinned edges, suggesting the flutter frequency will be higher as well. Higher frequencies are expected in this case, since the mass density of each shell in this system is significantly lower than the mass density of the single shell in models A and B. The regions of interest here are from $k=0$ to 5 for all $n$. The $2^{\text {nd }}$ and $3^{\text {rd }}$ axial modes are close in these regions, but the more important observation is that the separation between modes changes only a small amount when $1000 \mathrm{~N} / \mathrm{m}$ tension is applied. Recall that in this structural model, tension is only applied to the AKK shell. This result suggests that only tensioning the AKK will have little effect on the stability of the system.

The natural frequencies for model C-2 are given in Figure 13. When $k \geq 1$, the frequencies of the first three axial modes are initially higher than in model B-2, however, at large $k$ these modes in both models approach similar frequencies. Another similarity between the two models is that the $6^{\text {th }}$ and $7^{\text {th }}$ axial modes are close in frequency for several values of $k$. The regions of interest show that when tension is added, these frequencies shift only slightly, suggesting that the flutter boundary here may be less influenced by tension than in some of the other models. 


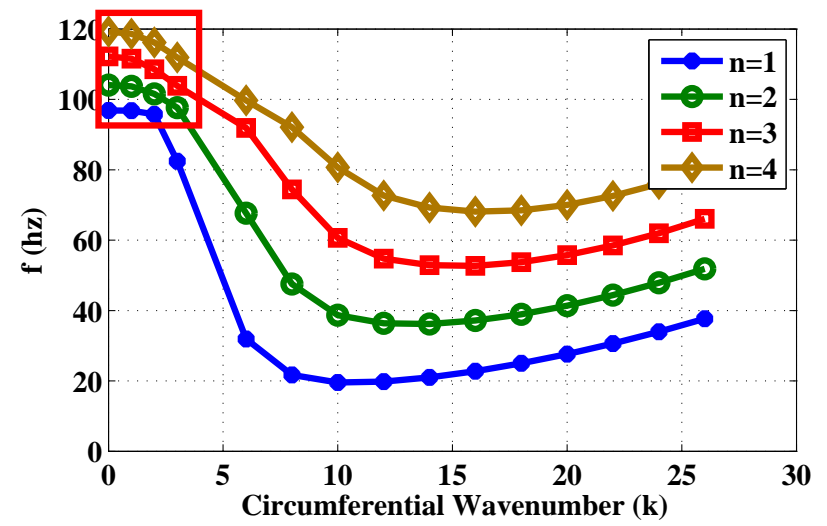

(a) $N_{y}^{a}=0$.

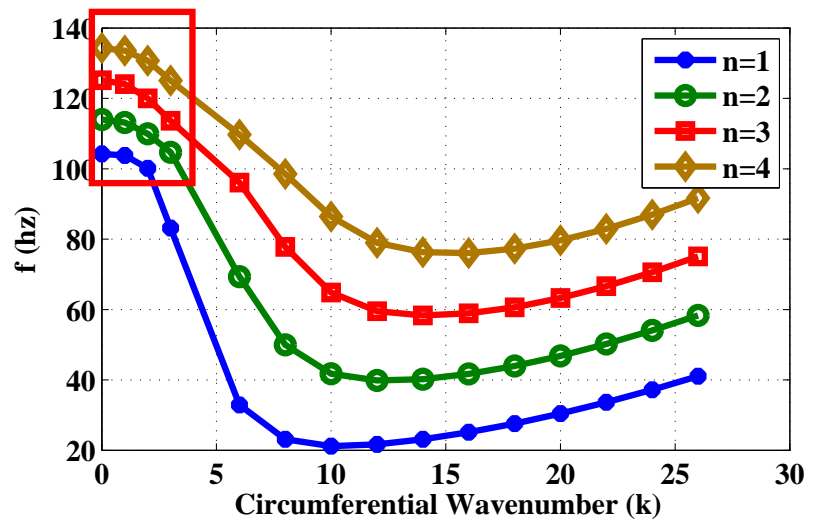

(b) $N_{y}^{a}=1000 N / m$.

Figure 8: Natural frequencies as a function of circumferential wavenumber, for model A-1.

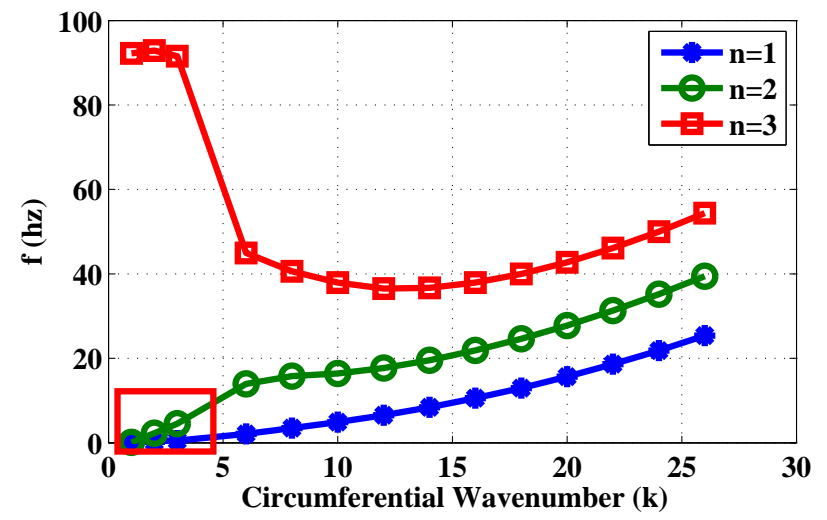

(a) $N_{y}^{a}=0$.

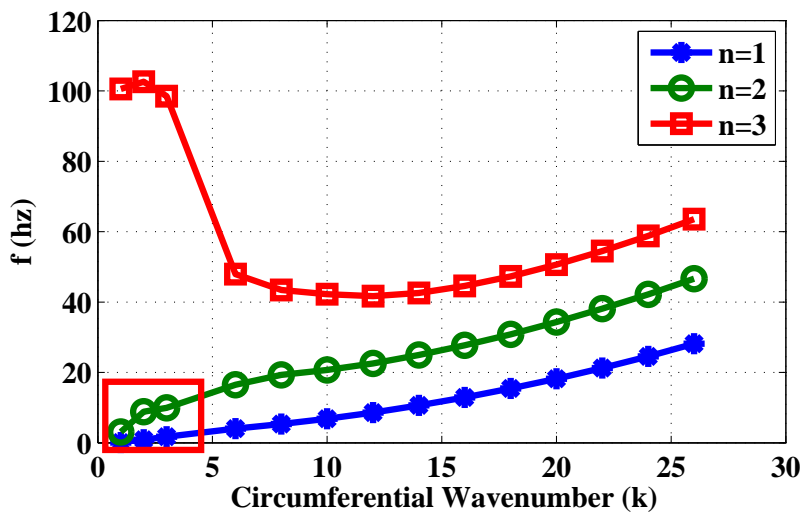

(b) $N_{y}^{a}=1000 \mathrm{~N} / \mathrm{m}$.

Figure 9: Natural frequencies as a function of circumferential wavenumber, for model A-2.

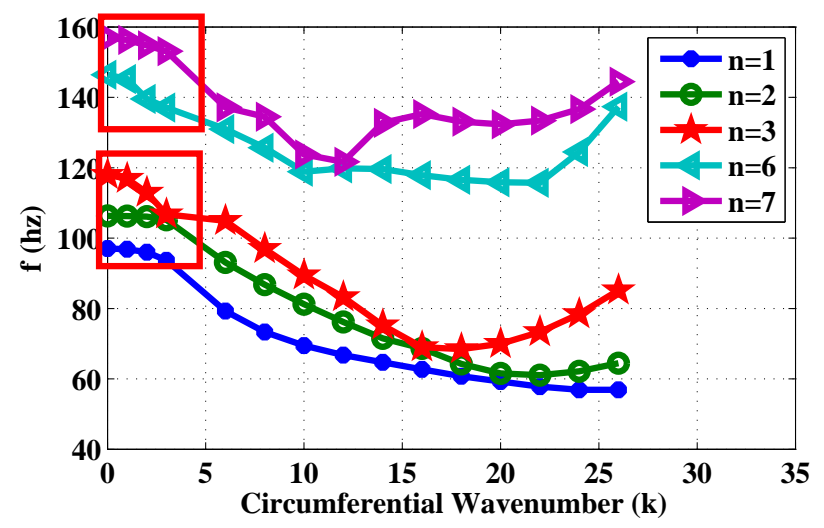

(a) $N_{y}^{a}=0$.

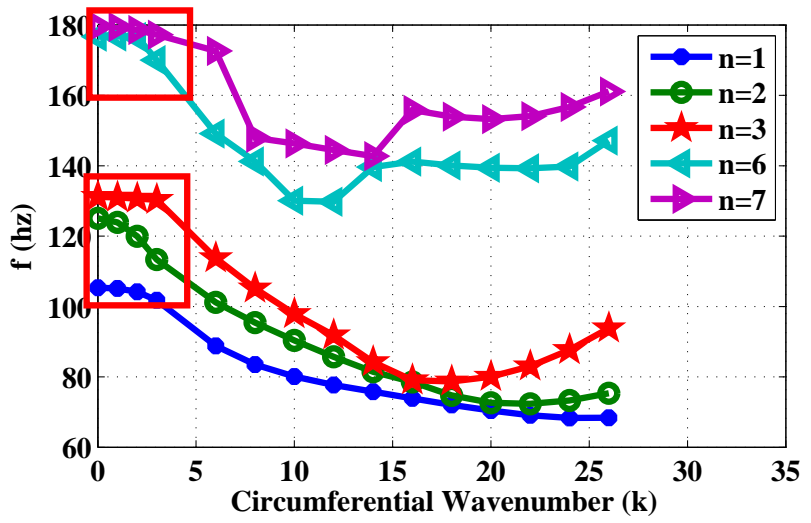

(b) $N_{y}^{a}=1000 \mathrm{~N} / \mathrm{m}$.

Figure 10: Natural frequencies as a function of circumferential wavenumber, for model B-1. 


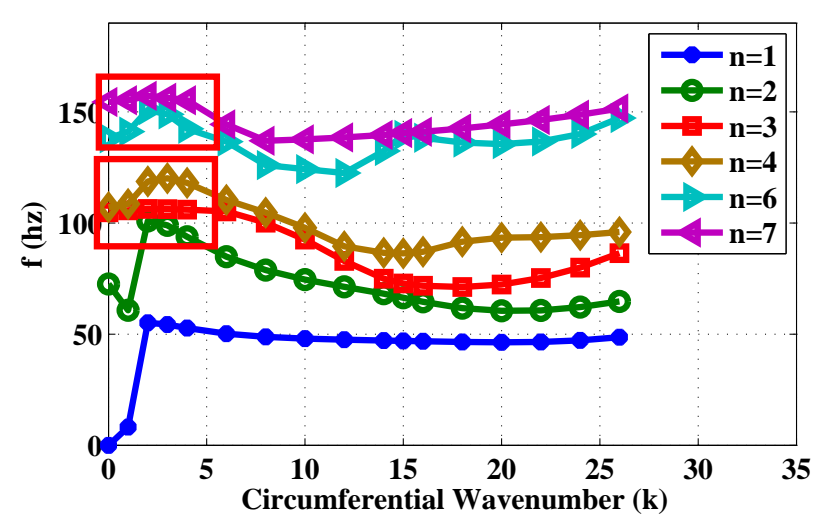

(a) $N_{y}^{a}=0$.

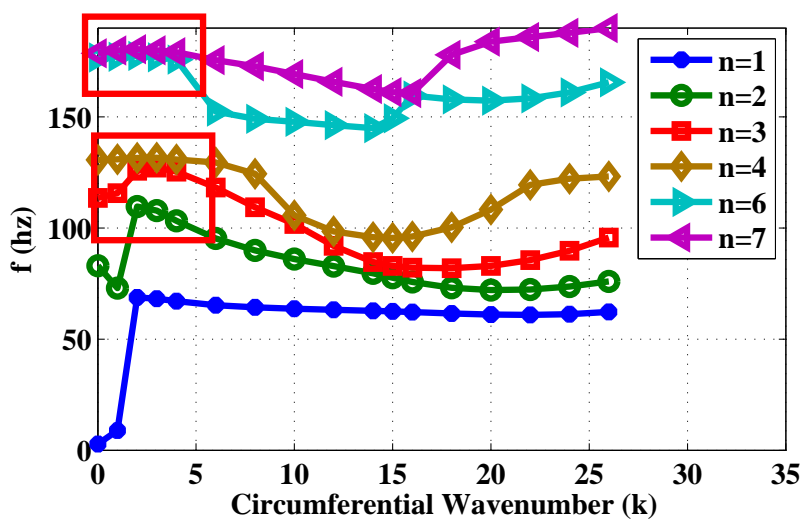

(b) $N_{y}^{a}=1000 \mathrm{~N} / \mathrm{m}$.

Figure 11: Natural frequencies as a function of circumferential wavenumber, for model B-2.

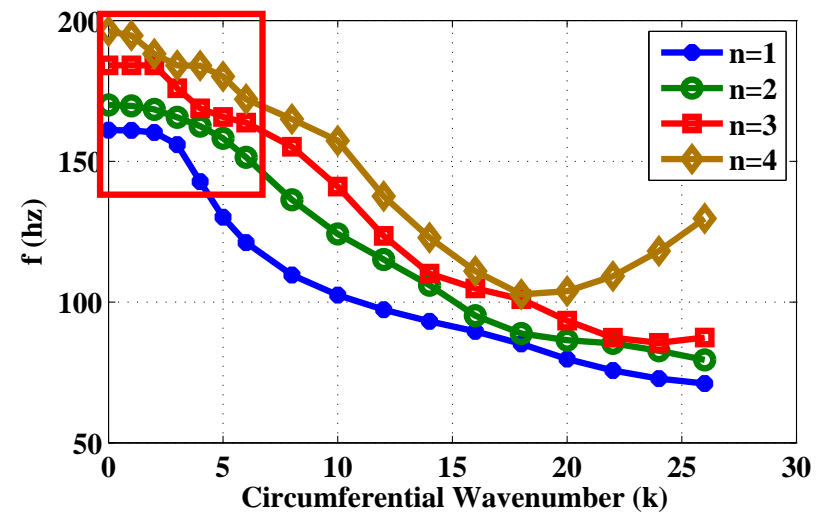

(a) $N_{y}^{a}(A K K)=0$.

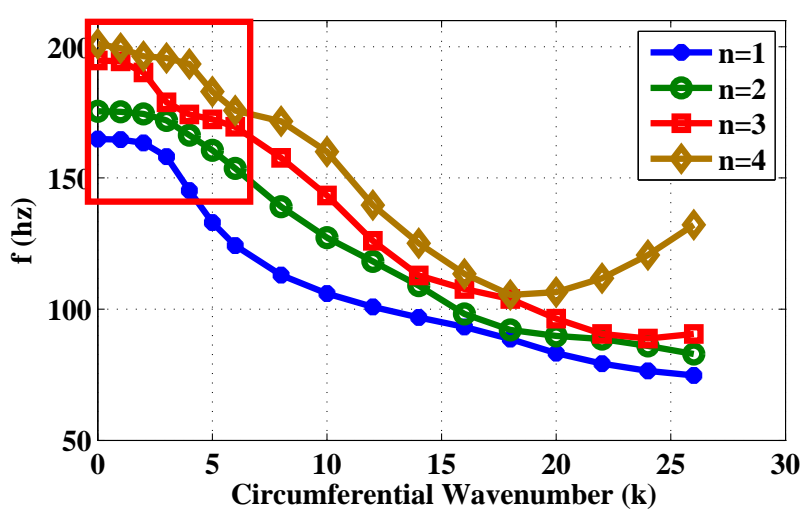

(b) $N_{y}^{a}(A K K)=1000 \mathrm{~N} / \mathrm{m}$.

Figure 12: Natural frequencies as a function of circumferential wavenumber, for model C-1.

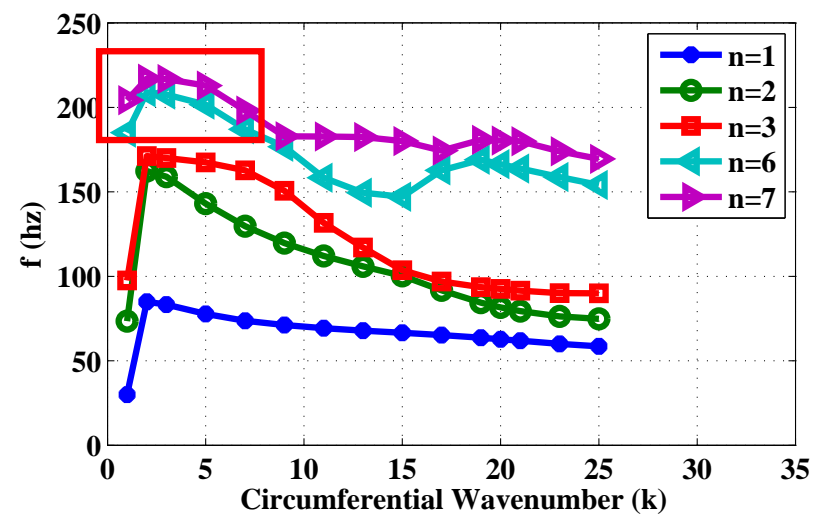

(a) $N_{y}^{a}(A K K)=0$.

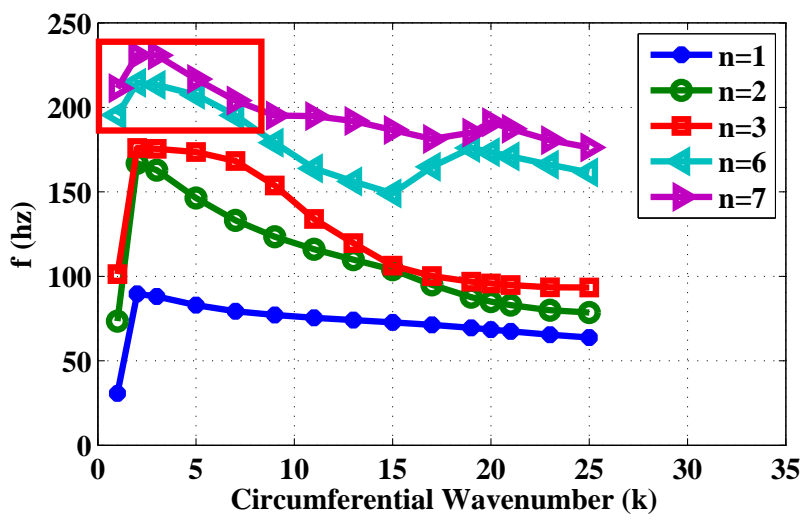

(b) $N_{y}^{a}(A K K)=1000 \mathrm{~N} / \mathrm{m}$.

Figure 13: Natural frequencies as a function of circumferential wavenumber, for model C-2. 


\section{V.C.2. Natural Mode Shapes}

The natural mode shapes in the circumferential direction are given in Fig. 14 for circumferential wavenumbers $0,1,2,3,5$, and 10 . These shapes are the same for all theoretical models in free-vibration as well as flutter. They are also independent of the shell geometry. Since the circumferential direction is decoupled from the axial direction, these shapes exist independent of deflections along the y-direction of the shell. The only axisymmetric mode is at $k=0$. In this case, all $v$ displacements are zero, and no waves propagate around the shell circumference. The $k=1$ mode shape may appear axisymmetric at first glance; however, the shell is slightly compressed in the shape of an ellipse in this case.

The natural mode shapes in the axial direction are dependent on the shell geometry, boundary conditions, and circumferential wavenumber. For conciseness, only the first three axial modes at $k=2$ and 3 will be provided here. Over this range of $k$, a variety of deflection behavior can be observed, i.e., rigid-body-like and pure bending modes. In addition, a given axial mode shape becomes less sensitive to changes in $k$ when $k$ is large (greater than 12).

The axial mode shapes for model A are given in Fig. 15. The mode shapes in each row have the same axial and circumferential mode numbers, while columns divide the results by the type of boundary conditions used. The effect of different boundary conditions is clearly demonstrated in this case, as model A-2 has several rigid-body-like modes, along with bending modes with nonzero deflections at the boundaries. Deflections in model A-1 are generally concentrated at the major end of the shell, where the shell is effectively less stiff due to the larger radius.

The axial mode shapes for model B are given in Fig. 16. At each elastic support location, deflection is constrained to near zero, which is a result of the relatively high elastic support stiffness in the nominal case. With such a high stiffness, the out-of-plane boundary conditions in models B-1 and B-2 are nearly the same, with in-plane displacements permitted at the boundaries only in the latter case. This leads to distinctly different deflection behavior for a given $n$ and $k$, with few exceptions (the $k=3, n=1$ mode shapes are similar). It is also observed that the $k=2, n=1$ mode shape for model B-1 is very similar to the corresponding mode shape for model A-1. Coincidentally, the elastic support at $\bar{y}=0.8$ in model B- 1 is located very close to a node for this mode in model A-1, thus having little effect on its shape.

The axial mode shapes for model $\mathrm{C}$ are given in Fig. 17. The solid and dashed lines in each sub-figure represent the Nextel and AKK shells, respectively. The separation between the two shells is equal to the nominal Pyrogel thickness. Since the Pyrogel stiffness is high, the two shells tend to deflect in unison at all points along the axial direction, which was also observed for the TPS plate models in Goldman and Dowell. ${ }^{5}$ Another result of the high Pyrogel stiffness is found by superimposing the mode shapes for the current model (C) onto those for the previous model (B). For nearly all modes, both the Nextel and AKK shells has nearly the same deflection shape as the single shell in model B (the shapes may have the opposite sign).
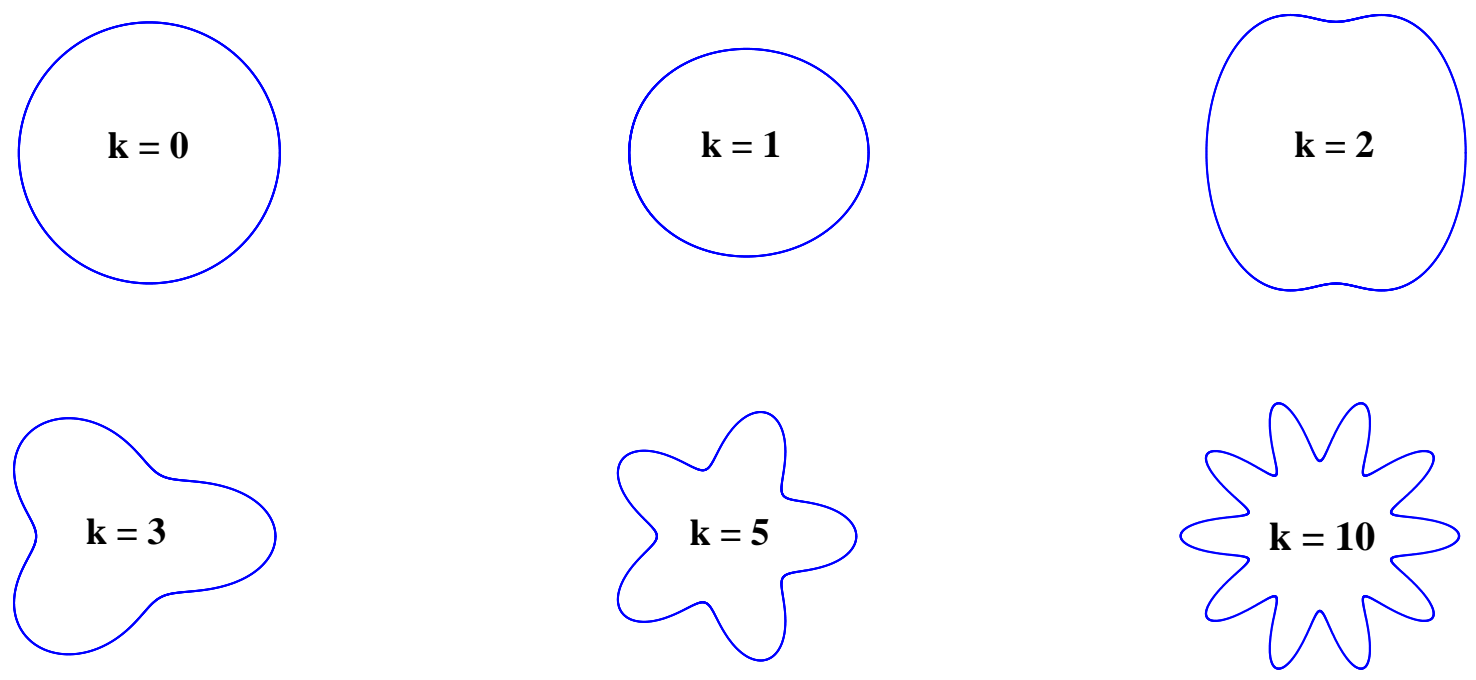

Figure 14: Circumferential mode shapes as a function of $k$. 

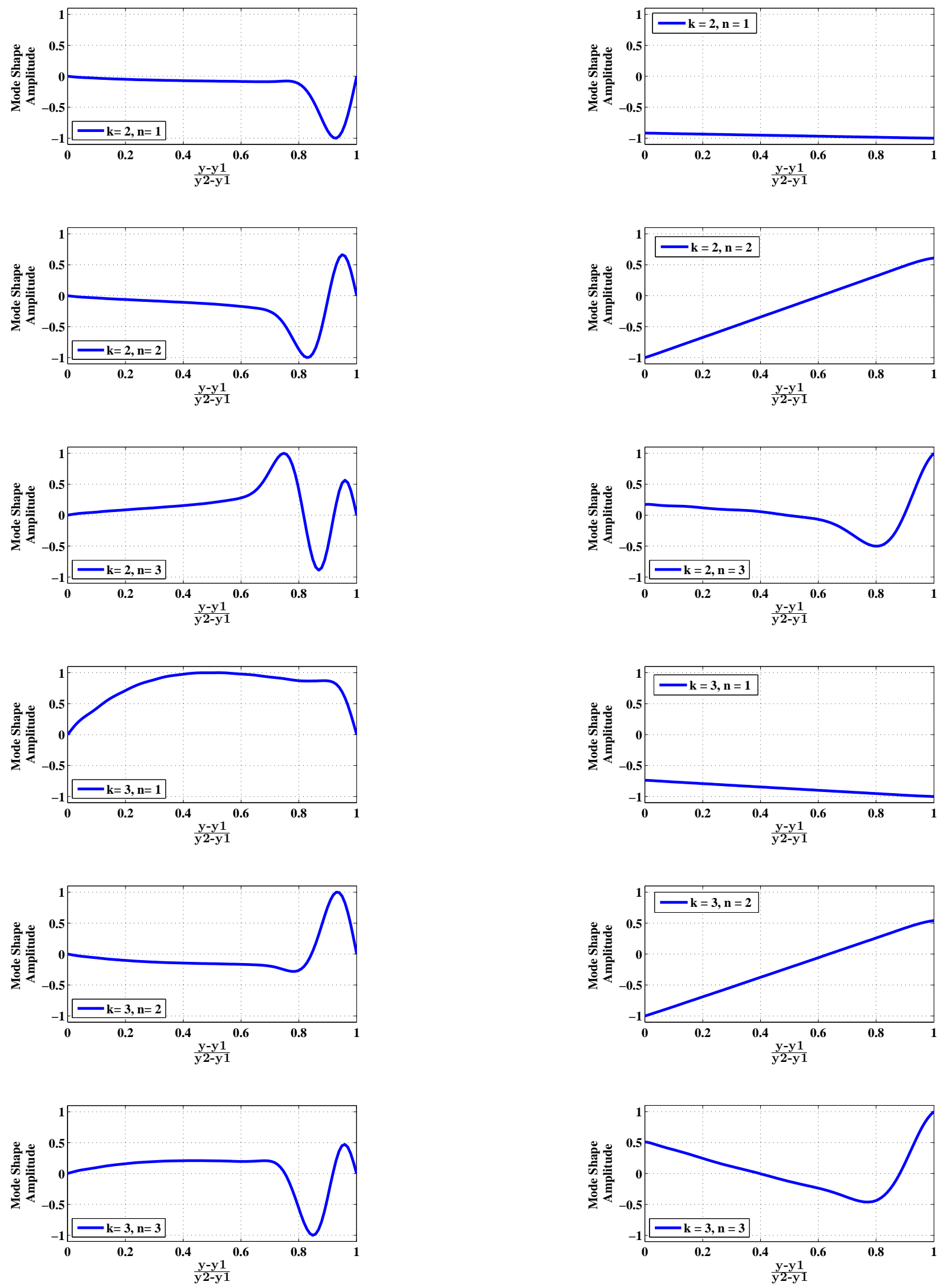

Model A-1

Model A-2

Figure 15: Selected axial mode shapes for model A. 

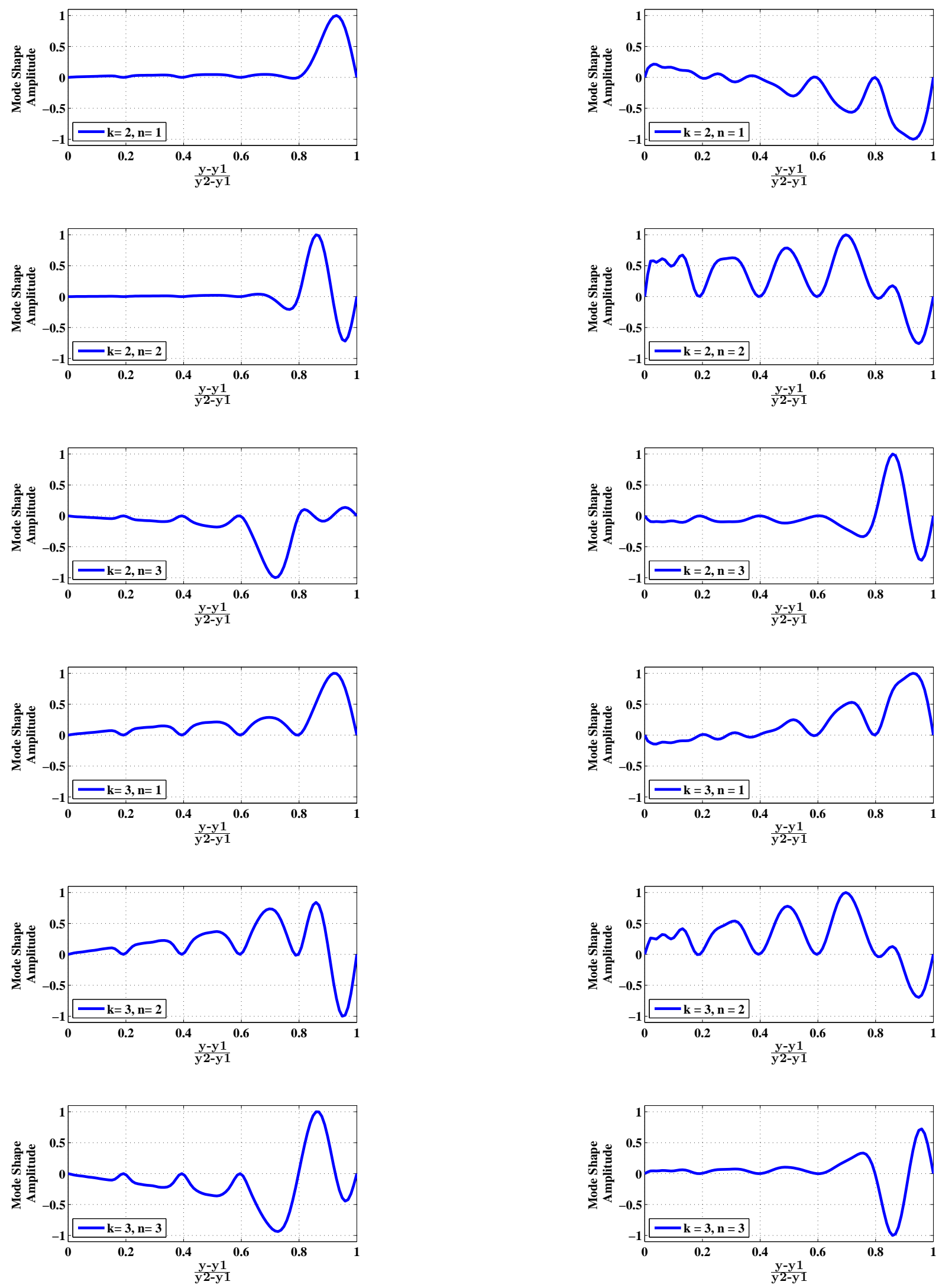

Model B-1

Model B-2

Figure 16: Selected axial mode shapes for model B. 

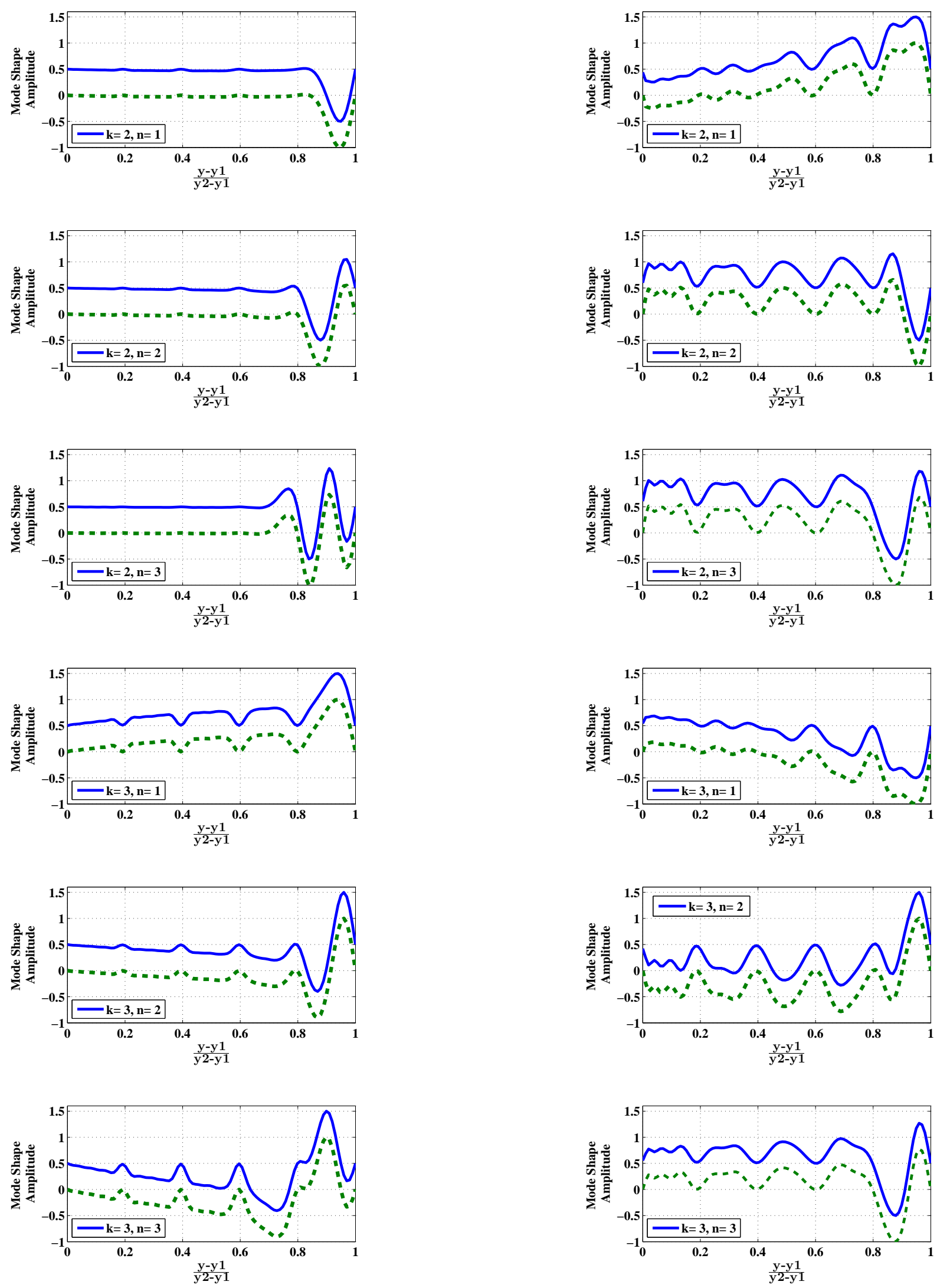

Model C-1

Model C-2

Figure 17: Selected axial mode shapes for model C. Solid line is the Nextel, and dotted line is the AKK. 


\section{V.D. Aeroelastic Stability}

In this section, the aeroelastic stability boundaries, flutter/divergence mode shapes, and a selection of parameter studies are presented. The nominal structural and aerodynamic parameters used in these computations were given earlier in Table 3 . In addition, aeroelastic results suggested that a simpler structural model with different structural parameters than those in Table 3 may be applicable in some cases, which will be discussed at the end of this section.

\section{V.D.1. Nominal Cases}

The aeroelastic results for each of the nominal cases in Table 4 are given in Table 5. Asymmetric flutter in three circumferential waves is observed for the single shell with pinned edges (A-1). Symmetric rigid-body divergence in zero circumferential waves is seen for the single shell with free edges (A-2). It was found that the rigid body modes in this model are unstable at any dynamic pressure infinitesimally greater than zero. However, when the piston theory curvature correction term ${ }^{19}$ is removed from the calculation, flutter in two circumferential waves occurs at significantly higher dynamic pressure. This is the only aeroelastic model that is significantly impacted by the curvature correction. Assuming that divergence at low $q$ is the instability mechanism, this aeroelastic model would not be a sufficient representation of the in-flight TPS configuration, and will not be discussed in further aeroelastic parameter studies.

A better representation of the in-flight TPS configuration is the elastically supported single shell model (B). In this case, flutter is observed for both types of boundary conditions. With pinned edges (B-1), the critical dynamic pressure for asymmetric flutter is only $8 \%^{*}$ higher than the pinned single shell without elastic supports (A-1). The flutter modes and frequencies are also identical. Since the only difference between the two cases is the addition of the elastic supports, a further investigation of the support properties is needed to explain why this is the case. A possible explanation is provided later in the discussion of the flutter mode shapes. Recall that in model B-2, Legendre polynomials coupled with the elastic supports result in elastically supported edges, with relaxed in-plane displacements. Here, the flutter modes and frequencies are similar to those in model B-1, yet the flutter dynamic pressure is significantly lower. Since in-plane displacements of the TPS is unrestricted on the HIAD vehicle, this flutter dynamic pressure may be more representative of what would be seen in-flight (under the single-shell assumption).

In model B-1, asymmetric hump mode flutter involving the $2^{\text {nd }}$ and $3^{\text {rd }}$ axial modes is observed. This instability occurs when two modes temporarily merge, resulting in negative system damping and flutter over a short range of dynamic pressures, then becoming stable again with increasing dynamic pressure. This is illustrated in Fig. 18a, which also indicates onset of coalescence flutter after decay of the hump mode. To determine which type of instability is likely to be observed in-flight, a small amount of structural damping is added to the system. While structural damping usually has a negligible effect on the flutter of shells, it may be significant in this special case. With $\zeta_{n}=0.01$, the region of positive system damping disappears. This is shown in Fig. 18b. Structural damping also prevents the $2^{\text {nd }}$ and $3^{\text {rd }}$ modes from completely merging, as indicated by the magnified region on the frequency sub-plot in Fig. 18b. Since the TPS materials are woven fabrics, they possess significant internal structural damping due to abrasion between the constituent fibers. The artificial addition of structural damping to the model is therefore justified, and the existence of the hump mode in flight is unlikely. It is assumed that flutter onset in in this model is caused by the coalescence mechanism, and the hump mode flutter boundary will be ignored. We note, however, that the existence of hump mode flutter in this model at nearly the same flutter dynamic pressure as model A-1 is not a coincidence, but rather an indicator of an instability related to the elastic supports. A discussion of the flutter mode shapes will help to clarify this result.

The flutter dynamic pressures and frequencies for model $\mathrm{C}$ are significantly greater than in any of the previous models. The increase in frequency is because the total mass of the single shell is distributed between two shells and the Pyrogel spring-layer. Even though the total mass of the system is the same, the effective mass of each shell here is much lower than the mass in the single shell models. Unlike the previous models, the case with pinned edges (C-1) flutters at relatively high $k$, while the elastically-supported case (C-2) flutters at relatively high $n$. Both cases undergo asymmetric flutter, indicating that the elastic supports have less influence in this system. This is further verified by the fact that the critical $n$ and $k$ for model C-1 are nearly the same as model A-1, the single shell. The greater flutter dynamic pressures and frequencies seen here also suggest that modeling the Nextel and AKK as separate shells is necessary if the TPS stitching density is low. However, if the stitching density is high, the TPS may behave more like a single-shell, in 
which case model B may be a better physical representation of the system.

\begin{tabular}{ccccccc}
\hline Model & $\boldsymbol{q}_{\boldsymbol{c r i \boldsymbol { i }}}(\boldsymbol{P a})$ & $\boldsymbol{n}_{\boldsymbol{c r i t}}$ & $\boldsymbol{k}_{\boldsymbol{c r i t}}$ & Instability type & $\boldsymbol{f}_{\boldsymbol{c r i \boldsymbol { t }}}(\boldsymbol{H z})$ & \# of modes \\
\hline A-1 & 6728 & 2,3 & 3 & Coalescence Flutter & 101 & 12 \\
\hline A-2 & $\rightarrow 0$ & 1 & 0 & Divergence & 0 & 8 \\
\hline \multirow{2}{*}{ B-1 } & $7329^{*}$ & 2,3 & 3 & Hump mode flutter & 106 & 46 \\
& 10866 & 1,3 & 0 & Coalescence flutter & 103 & 46 \\
\hline B-2 & 4392 & 2,3 & 0 & Coalescence Flutter & 106 & 46 \\
\hline C-1 & 27744 & 2,3 & 4 & Coalescence Flutter & 166 & 46 (each shell) \\
\hline C-2 & 34793 & 6,7 & 2 & Coalescence Flutter & 213 & 46 (each shell) \\
\hline
\end{tabular}

Table 5: Aeroelastic stability boundaries for the nominal cases.

*flutter boundary if $\zeta_{n}=0$.

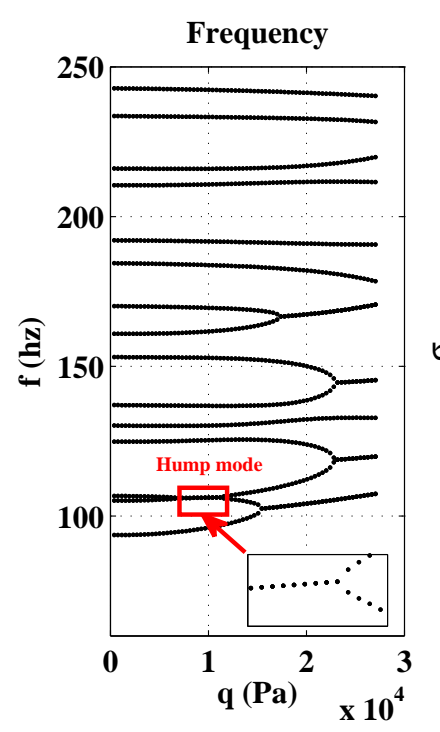

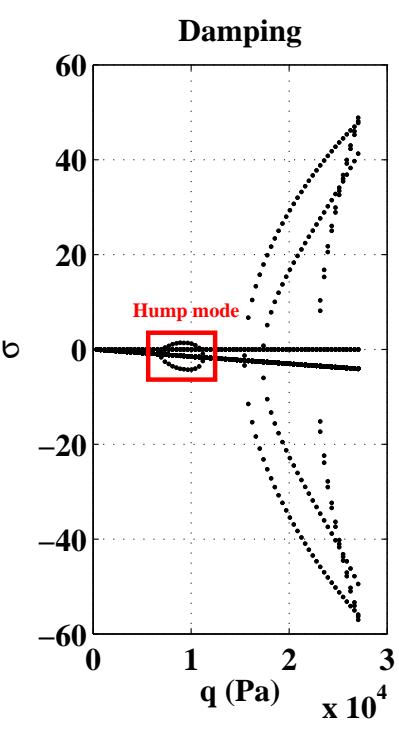

(a) $\zeta_{n}=0$.
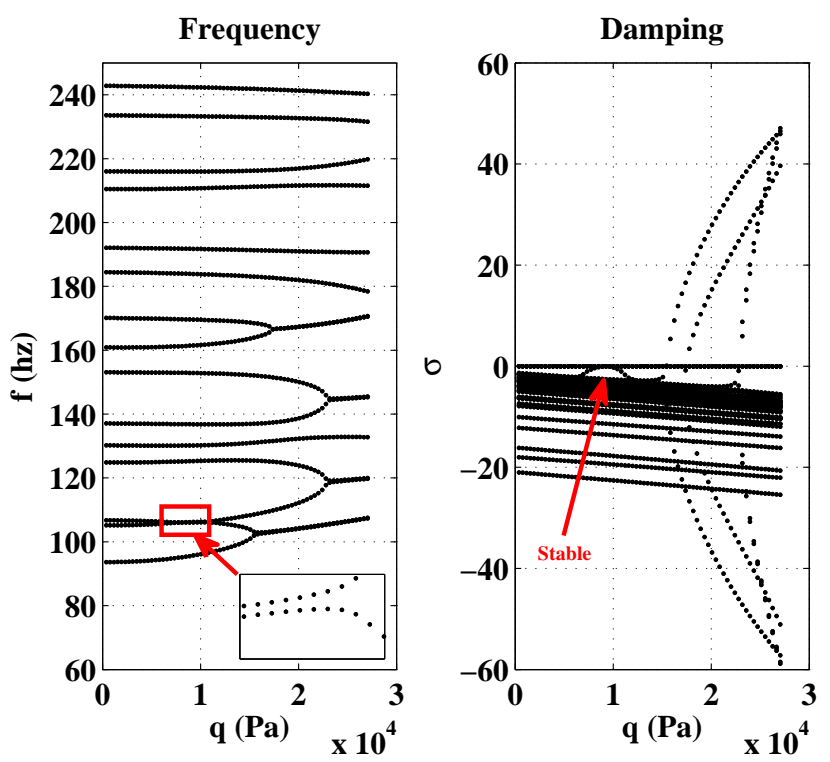

(b) $\zeta_{n}=0.01$.

Figure 18: Frequencies and system damping as a function of dynamic pressure and structural damping, illustrating the hump mode flutter instability in model B-1.

\section{Flutter $\mathcal{G}$ Divergence Mode Shapes}

The flutter and divergence mode shapes for all nominal cases in Table 5 are given in Fig. 19. The largest deflections are generally near the trailing edge (major end) of the shell(s), though the divergence mode shape in model A-2 is rigid-body-like. The effect of the elastic supports is demonstrated most clearly in the mode shapes for models B-1 and C-1, with deflections vanishing at each of the support locations. The large nominal stiffness of the supports is responsible for this result. In addition, deflection is observed in the sections between any two elastic supports, with amplitude increasing in each section closer to the major end. This result suggests that it may be possible to analyze only one section, instead of the entire shell. This simplification will be discussed further in section V.D.3.

The flutter mode shapes may also explain why the hump mode flutter dynamic pressure, critical axial modes, and critical circumferential modes for the elastically supported single shell (B-1) are nearly identical to that of the single shell alone (A-1). The second-to-last elastic support in model B-1, located at $\bar{y}=0.8$, is very close to an amplitude minimum in the flutter mode shape for model A-1, at approximately $\bar{y}=0.71$. Placing an elastic support exactly at this location in model A-1 has little effect on the flutter boundary, 
the flutter mode shape remains the same, and the flutter mechanism remains coalescence of the $2^{\text {nd }}$ and $3^{r d}$ axial modes. This similarity is demonstrated by the mode shape comparison in Fig. 19h. However, the second-to-last elastic support in model B-1 is located slightly ahead of this position, causing these modes to be closer in frequency than the corresponding modes in model A-1. This can be seen by comparing Fig. 10 to Fig. 8 at $k=3, N_{y}^{a}=0$. Due to the decrease in separation between these critical flutter modes, model B-1 exhibits hump mode flutter, with a similar deflection behavior to that of model A-1 in the region after the second-to-last elastic support. An interaction between the offset elastic support and aerodynamic stiffening may explain why hump mode becomes stable, transitioning to coalescence flutter at higher dynamic pressure (the hump mode is not sensitive to aerodynamic damping). Although hump mode flutter in-flight is unlikely due to the presence of structural damping, this analysis has demonstrated that the positioning of the elastic supports, and thus the toroids in the HIAD structure, is critical. If a toroid is located on the amplitude minimum of an aeroelastic mode, the TPS could flutter at significantly lower dynamic pressure than if the toroid were placed slightly fore or aft of this position.

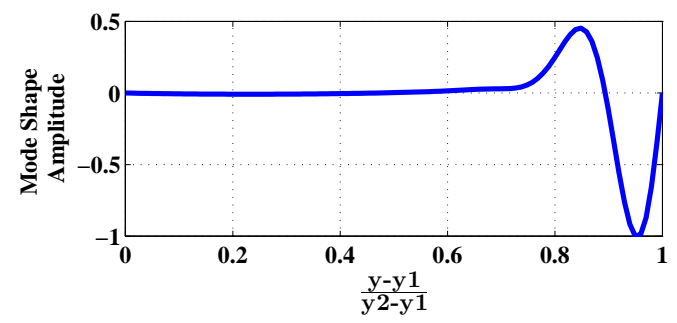

(a) Model A-1

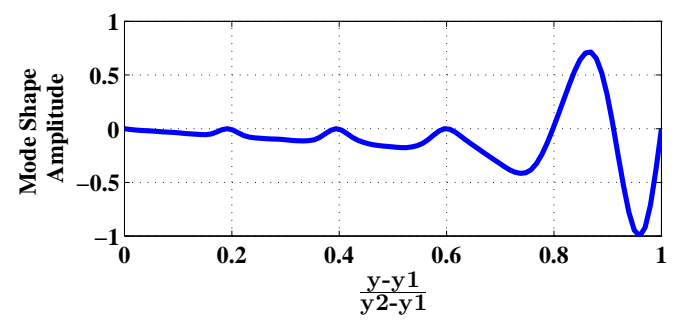

(c) Model B-1 (hump mode, $q_{\text {crit }}=7329$ Pa)

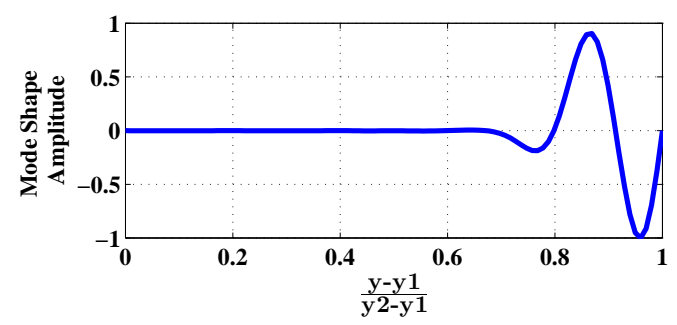

(e) Model B-2

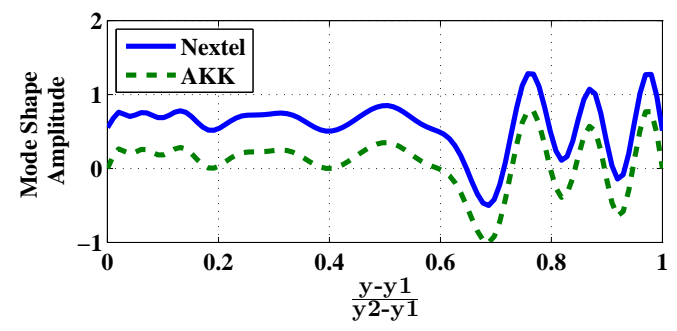

(g) Model C-2

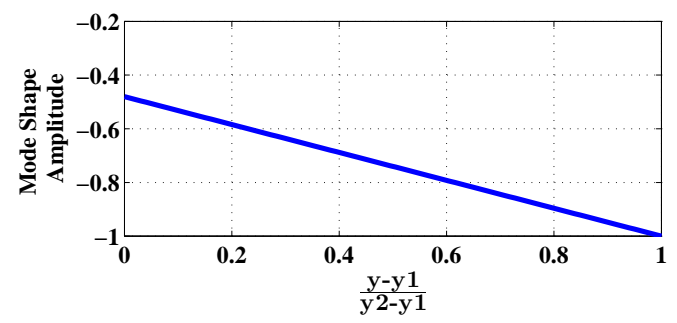

(b) Model A-2

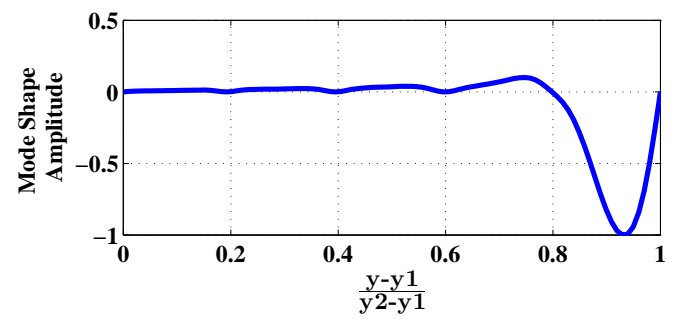

(d) Model B-1, $q_{\text {crit }}=10866 P a$

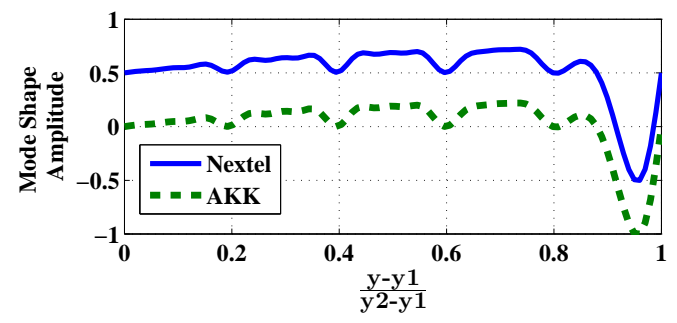

(f) Model C-1

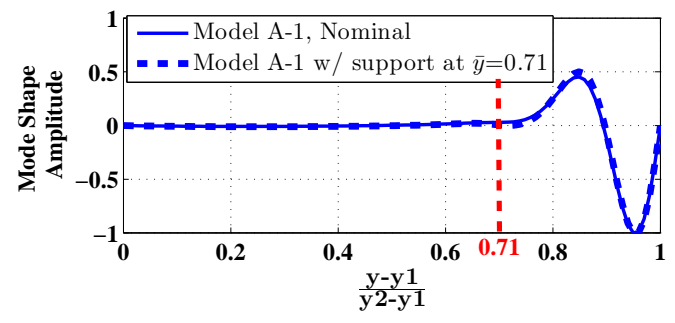

(h) Effect of a support at $\bar{y}=0.71$ in Model A-1.

Figure 19: Aeroelastic mode shapes in the axial direction for each TPS model. 


\section{V.D.2. Parameter Studies}

In this section, the influence of tension, $N_{y}^{a}$, shear modulus, $G_{y \theta}$, and elastic support stiffness, $K_{s}$ on aeroelastic stability is discussed. These quantities for the TPS in-flight configuration are presently unknown, but such parameter studies can be useful later when these data become available. Calculations for a wide range of parameter values may be beneficial in aeroelastically optimizing the TPS as well as the toroid substructure.

\section{Effect of tension}

The effect of tension on the aeroelastic stability of all models except A-2 is shown in Fig. 20 (tension has no effect on the divergence boundary of model A-2). The critical dynamic pressures, circumferential wavenumbers, axial modes, and frequencies are all given in sub-figures (a), (b), (c), and (d), respectively. The critical parameters in this case all refer to flutter. It is observed that the flutter dynamic pressure initially increases linearly with tension for all models except B-1, but this trend does not hold for higher tension values. For example, the flutter dynamic pressure for model C-1 starts to level off at $N_{y}^{a}=1000 \mathrm{~N} / \mathrm{m}$. In this case, there is transition from $k_{c r i t}=4$ to $k_{c r i t}=3$. Transitions in the critical circumferential and axial modes are seen for all other models, and may be responsible for the non-linear relationship between tension and flutter dynamic pressure. A similar trend is observed in the flutter frequency, which is directly correlated to the behavior of the critical axial modes.

\section{Effect of Shear Modulus}

As previously mentioned, the TPS materials are relatively inextensible in tension but very flexible in shear. It is therefore of great interest to examine the effect of decreasing shear modulus on the flutter boundary of the aeroelastic models. These results are shown in Fig. 21. Decreasing the shear modulus with respect to the Young's modulus initially increases the flutter dynamic pressure for most models when $G_{y \theta} / E_{y} \leq 10^{-2}$, after which the flutter dynamic pressures generally decrease. Little influence is seen for models B-1 and B-2. It may be that the circumferential elastic supports provide enough stiffness to mitigate the reduction in shell stiffness in this case. In models $\mathrm{C}-1$ and $\mathrm{C}-2$, the shear modulus is decreased by the same amount in both shells, resulting in a rapid decrease in the flutter dynamic pressure. Since the outer Nextel shell is not directly connected to the elastic supports, it may be more sensitive to decreasing shear modulus than AKK shell. The difference between the flutter boundaries for model B and model C suggests this is likely the case. The flutter frequency for all models also decreases, though not smoothly. As the shear modulus approaches zero, the flutter frequencies decrease to just a few Hertz, and the critical circumferential wavenumbers in all cases converge to 1.

\section{Effect of Elastic Support Stiffness}

The nominal stiffness of the elastic supports is large, since the toroid substructure on the HIAD is rather inflexible when inflated. Therefore, very low elastic support stiffness may not be physically representative, but an analysis of these configurations may help explain some of the earlier results. In Fig. 22, both flutter and divergence is observed, and lower $K_{s}$ also lowers the critical dynamic pressure for all models except C-1. This is an unusual case, since the flutter modes and frequencies as a function of $K_{s}$ are relatively constant. A possible hypothesis that may explain this behavior is that the boundaries are always pinned, irrespective of the decreasing support stiffness. A similar theory may be used to explain why model C-2 has the opposite result. The boundaries in this case have the same out-of-plane stiffness as the elastic supports, so decreasing $K_{s}$ by a large enough amount should cause the boundaries to approach the free-free condition. Indeed, at $K_{s} / K_{s, \text { nominal }}=10^{-5}$, divergence is observed, rather than flutter. The same divergence boundary is seen for model B-2. Recall that divergence was the only instability observed for model A-2, the free-free shell. Decreasing $K_{s}$ in model B-1 also results in some noteworthy behavior. At $K_{s} / K_{s, \text { nominal }}=10^{-1}, k_{c r i t}$ and $n_{\text {crit }}$ transition to that of the hump mode flutter boundary discussed earlier in section V.D.1 (with $k_{\text {crit }}=3$ and $n_{\text {crit }}=2,3$ ). In this case however, the decreased stiffness results in coalescence flutter, rather than hump mode flutter. Recall that coalescence flutter involving these same critical modes was observed for the single shell in model A-1. It is therefore unsurprising that the flutter dynamic pressure for Model B-1 changes only slightly with respect to decreasing $K_{s}$, rapidly approaching the flutter boundary of the single-shell, shown as the dashed line in Fig. 22a. 


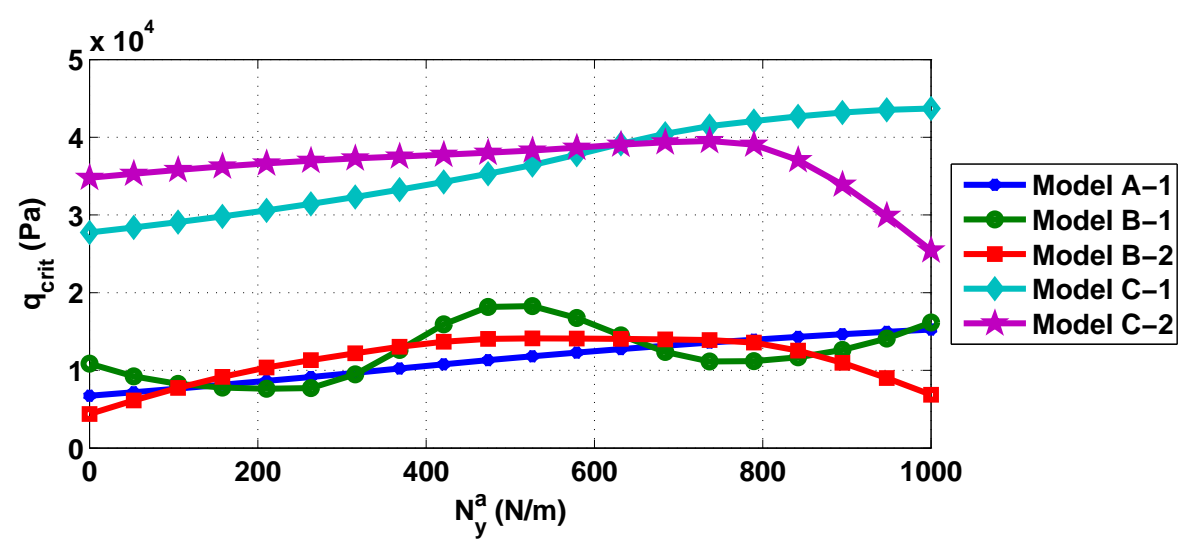

(a) Critical dynamic pressure as a function of tension.

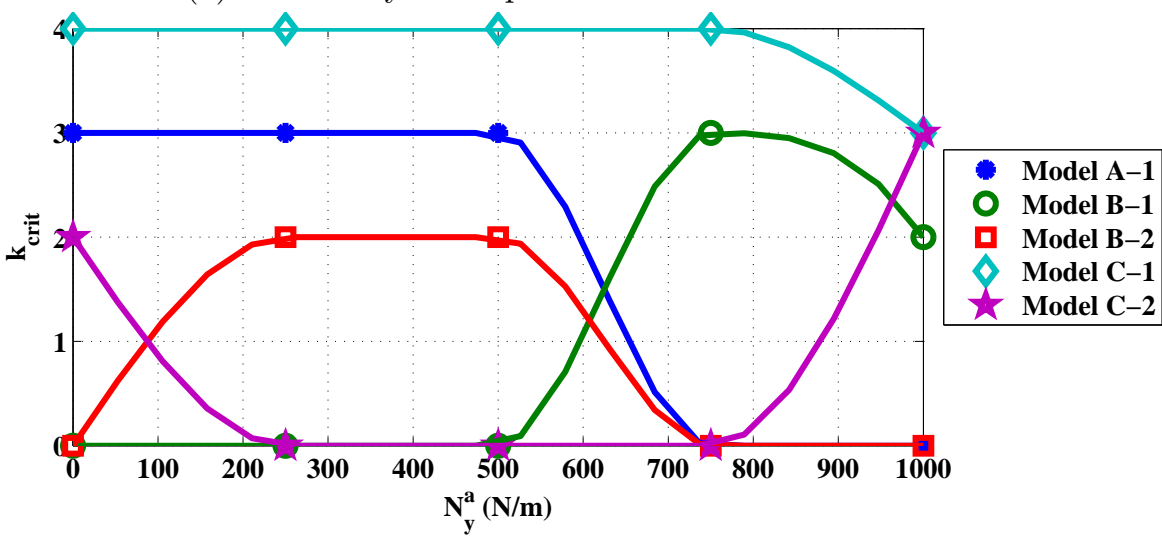

(b) Critical circumferential wavenumber as a function of tension.

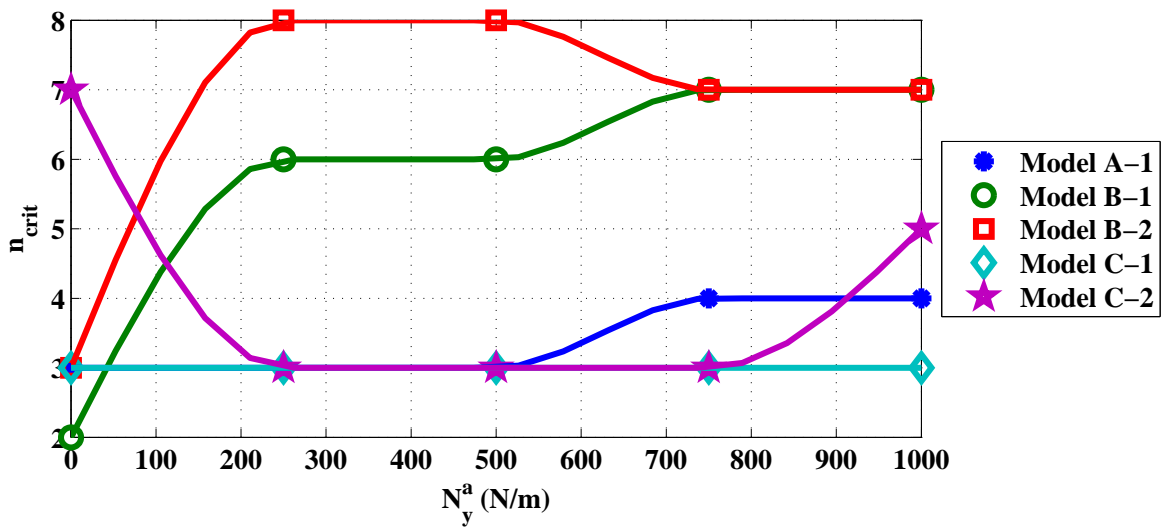

(c) Critical modes as a function of tension.

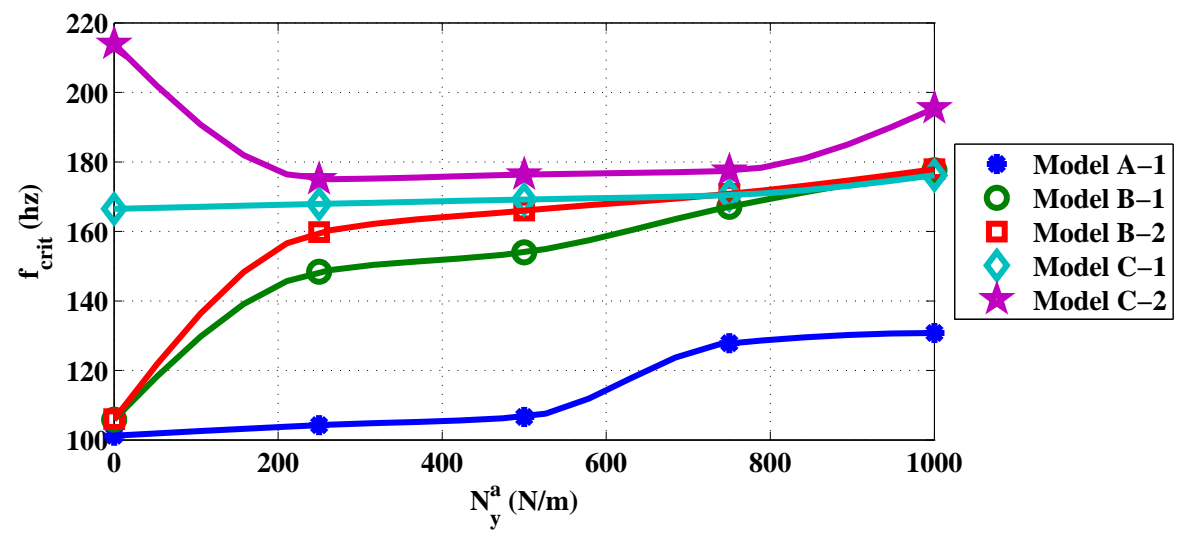

(d) Critical frequency as a function of tension.

Figure 20: Effect of tension $\left(N_{y}^{a}\right)$ on the flutter boundary for all models except A-2. 


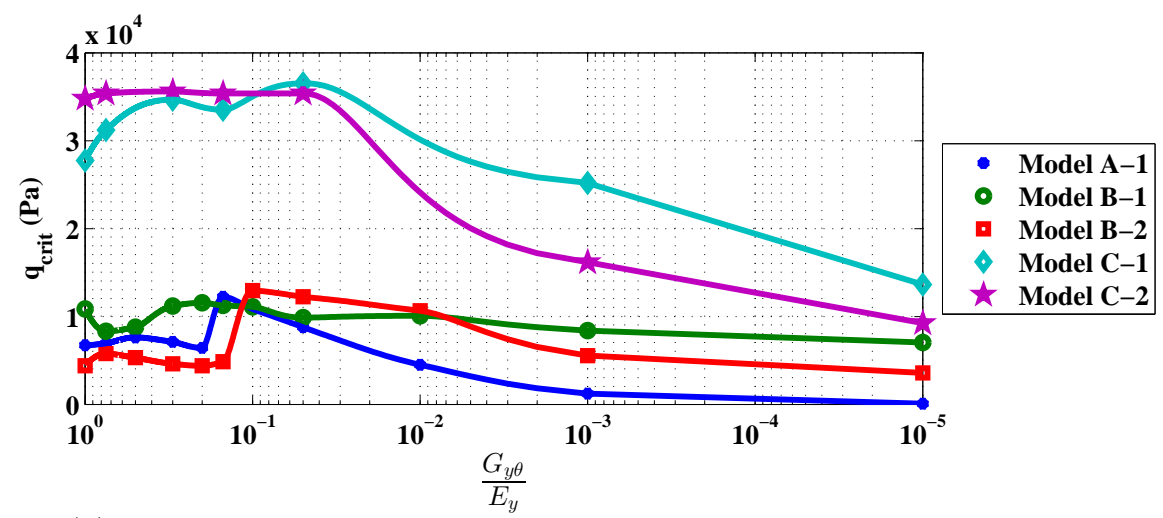

(a) Critical dynamic pressure as a function of in-plane shear stiffness.

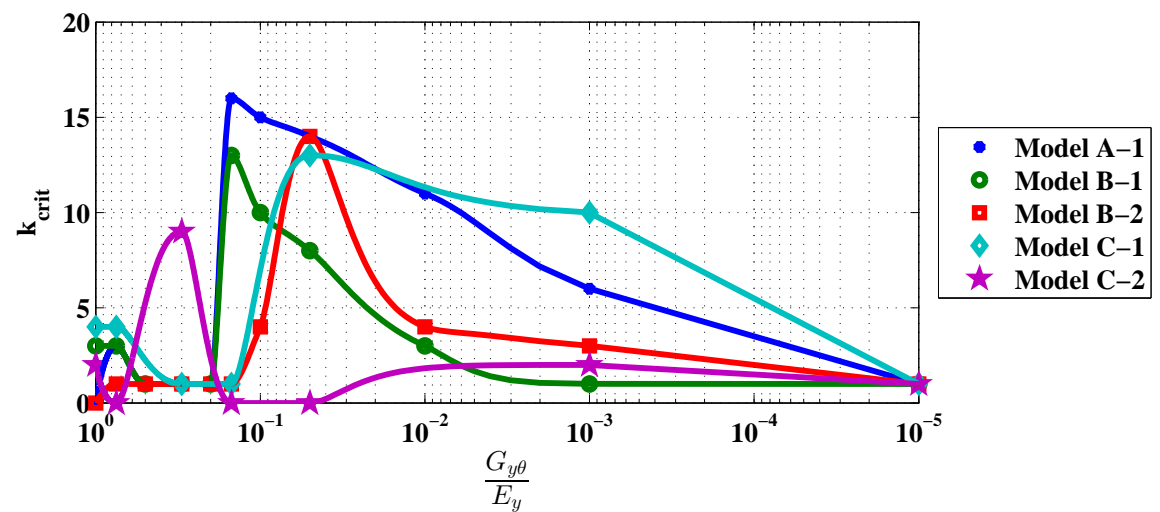

(b) Critical circumferential wavenumber as a function of in-plane shear stiffness.

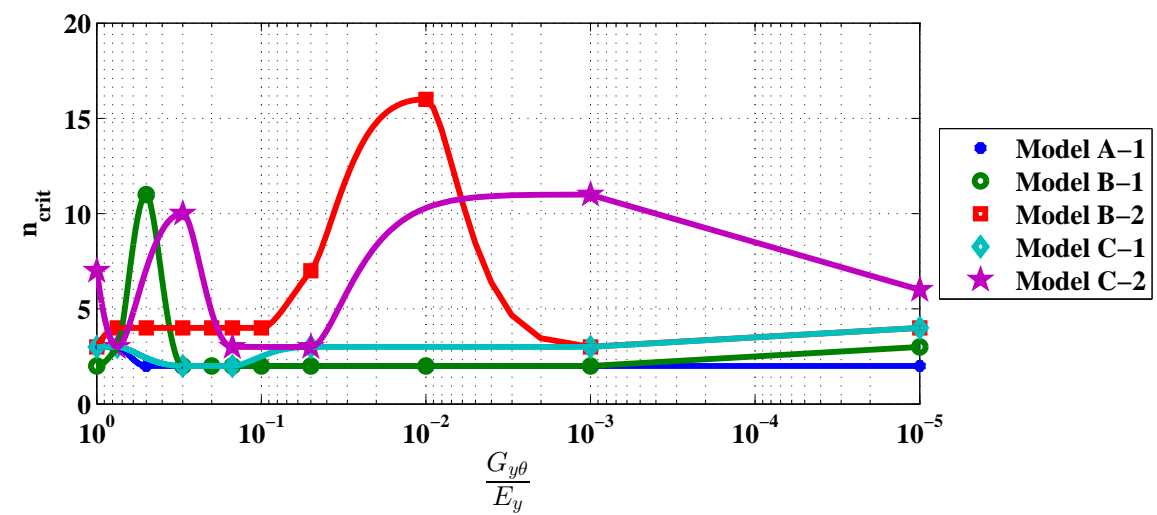

(c) Critical modes as a function of in-plane shear stiffness.

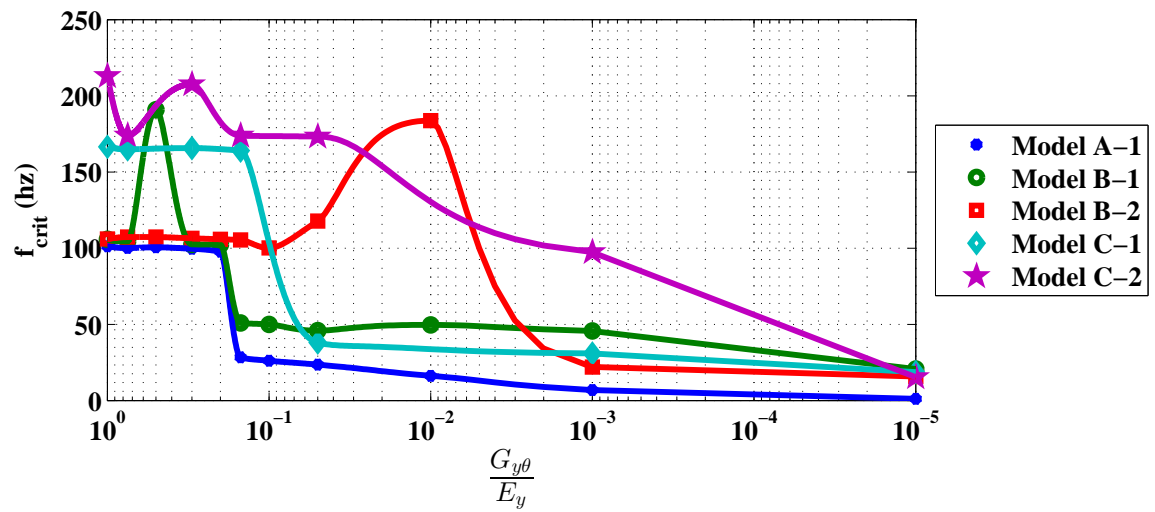

(d) Critical frequency as a function of in-plane shear stiffness.

Figure 21: Effect of in-plane shear stiffness on the flutter boundary for all models except A-2. 


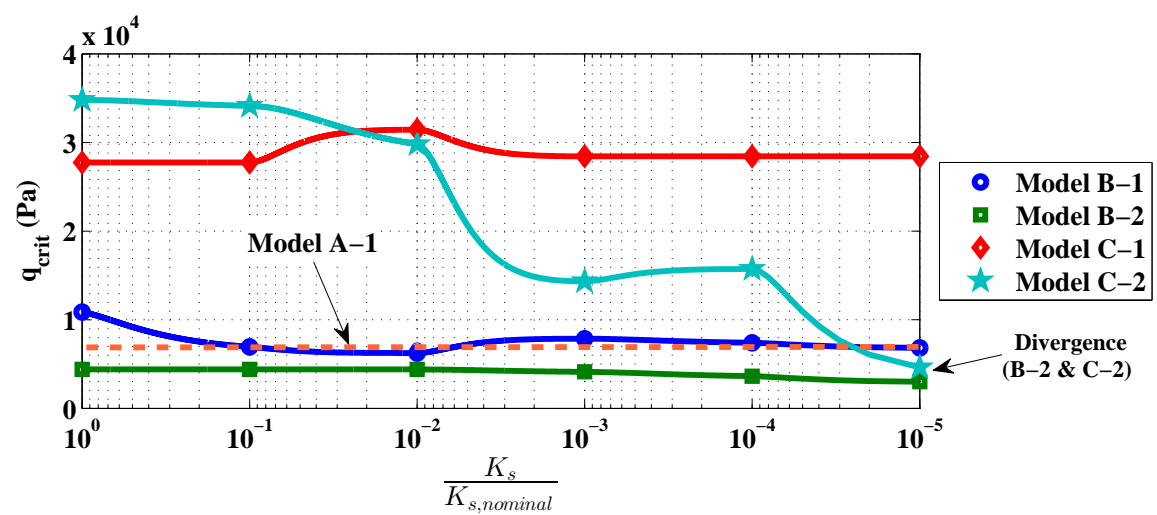

(a) Critical dynamic pressure as a function of elastic support stiffness.

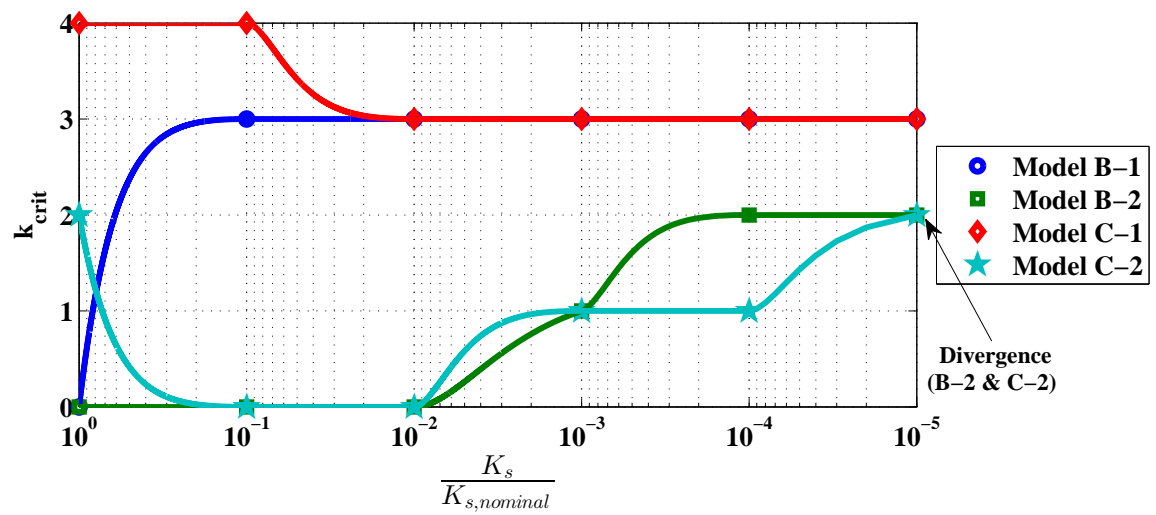

(b) Critical circumferential wavenumber as a function of elastic support stiffness.

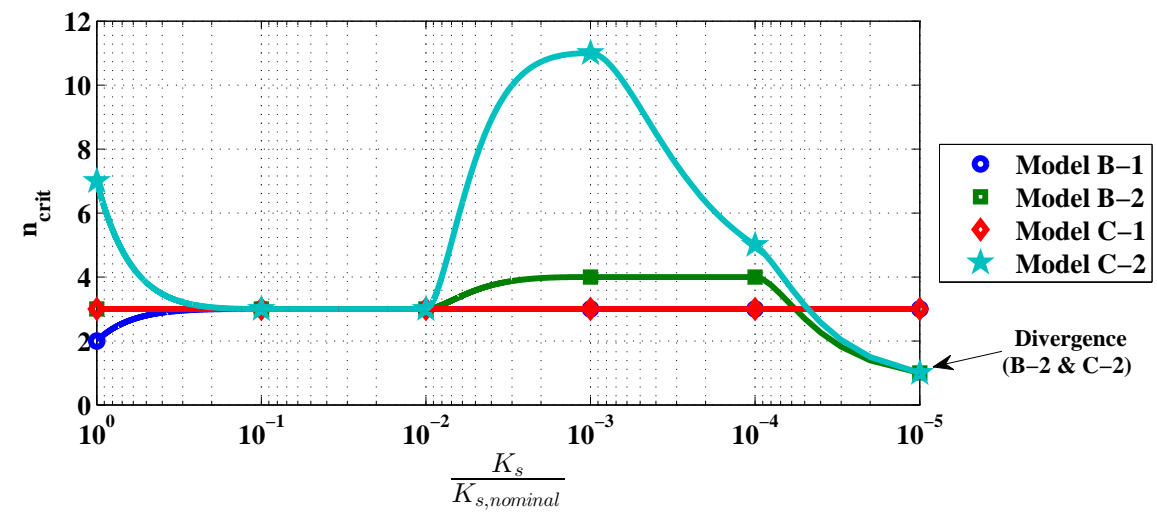

(c) Critical modes as a function of elastic support stiffness.

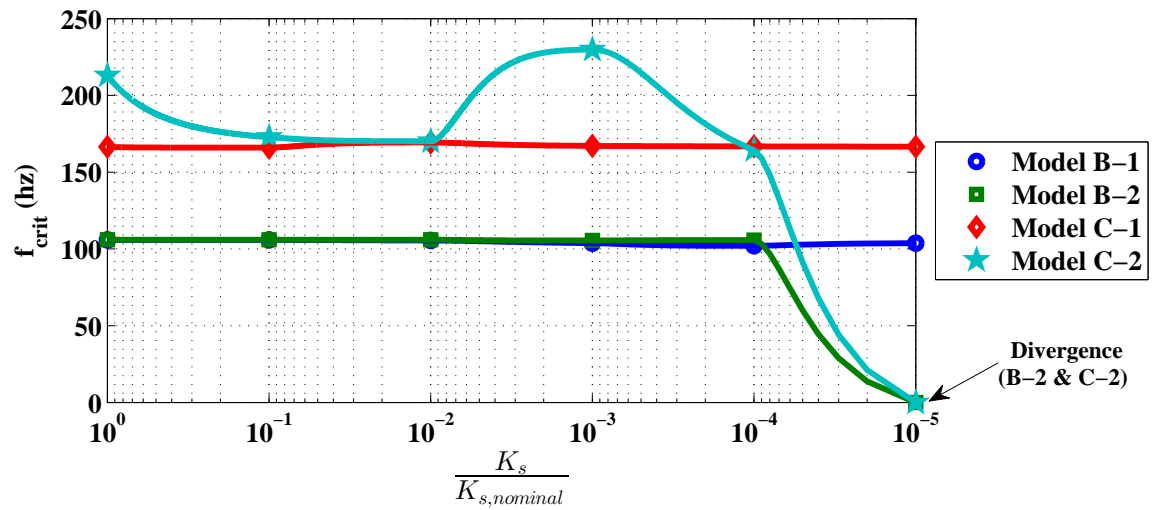

(d) Critical frequency as a function of elastic support stiffness.

Figure 22: Effect of elastic support stiffness on the flutter/divergence boundaries for models B and C. 


\section{V.D.3. Structural Simplification of the Elastically Supported Models}

Analysis of the flutter mode shapes in section V.D.1 indicated that some of the models with six circumferential elastic supports could be reduced to simpler aeroelastic systems. Deflection is observed in each section between any two elastic supports, with the amplitude increasing in each section closer to the major end of the shell. Not all models exhibit this behavior, but these results suggest that a shell consisting of only the largest section, that between the last two elastic supports may have similar aeroelastic stability as a complete shell with all six elastic supports. An example of this simplified system for the three-layer shell model is illustrated in Fig. 23.

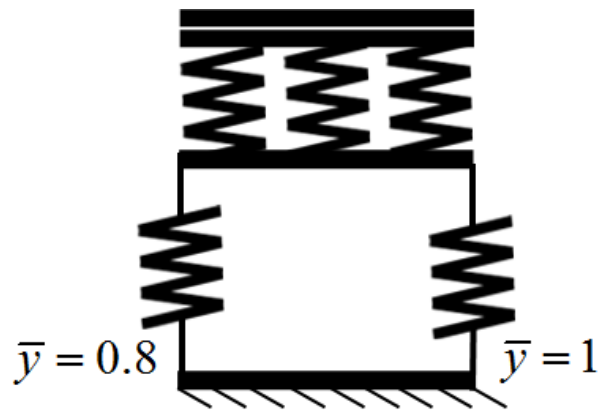

Figure 23: Single-section model of the elastically-supported three-layer shell (cross-section view).

Flutter dynamic pressures have been computed for each elastically-supported model, considering only the section between $\bar{y}=0.8$ and $\bar{y}=1$. In models B-1 and C-1, pinned displacements are still enforced at the boundaries of the section, such that the elastic supports in Fig. 23 are rigid. The supports in models B-2 and C-2 have the nominal stiffness. These results are given in Table 6 , along with the previously calculated flutter boundaries for comparison. Best agreement is seen for models B-2 and C-1. There is a substantial discrepancy for models B-1 and C-2, though the hump mode flutter boundary in model B-1 is closer to the largest-section result. Recall that the hump mode occurs due to the proximity of the second-to-last elastic support to the amplitude minimum in the aeroelastic mode of the single shell. The single-section approach breaks down in this case, because the modes of the entire shell cannot be resolved. This method could still be used in preliminary modeling or design studies, since all results are consistent within $50 \%$.

\begin{tabular}{ccc}
\hline & \multicolumn{2}{c}{ Critical Dynamic Pressure $(\mathrm{Pa})$} \\
\hline Model & Complete shell & Largest section \\
\hline B-1* & 7329 & 6011 \\
B-1 & 10866 & 6011 \\
B-2 & 4393 & 4624 \\
C-1 & 27744 & 27350 \\
C-2 & 34796 & 25432 \\
\hline
\end{tabular}

Table 6: Comparison of the flutter dynamic pressures for the shell section between $\bar{y}=0.8$ and $\bar{y}=1$.

*hump mode flutter boundary, $\zeta_{n}=0$

\section{Concluding Remarks}

A theoretical aeroelastic evaluation of the generation 1 thermal protection system on the NASA Hypersonic Inflatable Aerodynamic Decelerator has been presented in this paper. Conical shell theory and piston theory aerodynamics were used to develop several linear aeroelastic models for the TPS in-flight configuration. The simplest model, A, considered the TPS as a single shell, and the toroid substructure on the HIAD was ignored. The next model, B, also considered the TPS as a single shell, but included the effect of the toroid substructure by adding several circumferential elastic supports along the shell middle-surface. The most elaborate model, C, considered all three TPS layers, with the Nextel and AKK as individual shells separated by a Pyrogel spring-layer. The circumferential elastic supports were also included. In all models, 
two types of modal functions were considered in the expansions of shell displacements. Sinusoidal modal functions resulted in fixed displacements at the shell boundaries, while Legendre polynomials resulted in free or elastically-supported edges, depending on the model. The Lagrangian was formulated in terms of three generalized coordinates and the Rayleigh-Ritz method was used to derive the equations of motion. Aeroelastic solutions were obtained by writing the equations as an eigenvalue problem, and observing the behavior of the eigenvalues with increasing flow dynamic pressure.

Prior to analyzing aeroelastic stability, the natural modes of vibration were determined for a set of nominal cases. The frequencies of the axial modes depend on the circumferential wavenumber, $k$, as well as the amount of tension applied at the shell edges. The separation between frequencies for a given set of axial modes was also sensitive to tension. Aeroelastic stability boundaries were calculated for the same set of nominal cases. Both symmetric and asymmetric flutter was observed, depending on the model. The single shell experienced asymmetric flutter in three circumferential waves, while the elastically supported single shell experienced symmetric flutter in zero circumferential waves, at a significantly higher dynamic pressure. In the latter case, hump mode flutter also occurred at a lower dynamic pressure, near that of the former case. This indicated that an elastic support had been placed near an amplitude minimum of the aeroelastic mode for the single shell, thereby decreasing the influence of the support stiffness and lowering the flutter boundary. While the hump mode was found to be stabilized with a small amount of structural damping, moving the elastic support closer to this amplitude minimum resulted in coalescence flutter that did not disappear with structural damping added. These results suggest that the locations of the toroids in the HIAD substructure may be critical to the aeroelastic performance of the TPS.

The influence of varying tension, shear modulus, and elastic support stiffness on aeroelastic stability was also studied. It was found that the flutter dynamic pressure did not increase uniformly with tension, due to transitions between critical circumferential and axial modes. This suggests that large tension applied to the TPS in-flight may not necessarily result in the highest flutter boundary. Note that these calculations are subject to the limitations of the linear membrane theory approximation (see Eq. 6), so a nonlinear analysis is necessary to verify the findings. The orthotropic nature of the TPS materials was considered by reducing the in-plane shear modulus with respect to the Young's modulus. This generally had a destabilizing effect, especially when the nominal shear modulus was decreased by more than two orders of magnitude. Reducing the elastic support stiffness generally reduced the critical dynamic pressure, and a low enough stiffness resulted in divergence for the models with elastically supported boundaries. Lowering the nominal stiffness by less than one order of magnitude also resulted in a transition from hump mode flutter to coalescence flutter in the elastically-supported single shell, with a critical dynamic pressure near that of the single shell alone. The results are less pertinent to the in-flight performance, since the toroid substructure is nearly rigid when completely inflated.

In the aeroelastic mode shapes for the models with elastic supports, deflections were largest in amplitude between the two elastic supports closest to the major end of the shell, but smaller amplitude deflections were also observed in the sections between elastic supports along the entire shell length. This suggested that a simpler aeroelastic analysis could consider only a section of the shell between the last two supports. A comparison of the results from this "single-section" approach to the results of the nominal cases with all six supports indicated that the method could be used as an initial approximation, since good agreement was seen in a subset of cases, and fair agreement for the remaining cases.

To determine which (linear) aeroelastic model is most representative of the TPS in-flight, it is necessary to study further the TPS layup configuration. If the stitching density between the Nextel and AKK layers is low, the elastically-supported three-layer shell model may be more appropriate, since the individual layers are considered separately. However, if the stitching density is very high, the TPS will likely behave as a single shell, such that elastically-supported single shell may more representative. The flutter dynamic pressure in the latter case is significantly lower than in the former, according to linear theory. In part II of this report series, a nonlinear shell model will be studied, allowing the static pressure differential and tension to be included as non-conservative virtual work, rather than membrane energy. Large static deflections are expected in flight, the effects of which can be accounted for in nonlinear theory. The nonlinear "unidirectional" interaction between the toroid substructure and the TPS will be also considered. To study the more realistic case of low Mach number supersonic flow, piston theory aerodynamics will be extended using the method of Dowell and Bliss. ${ }^{21}$ 


\section{Appendix}

\section{Shell Coordinate System}

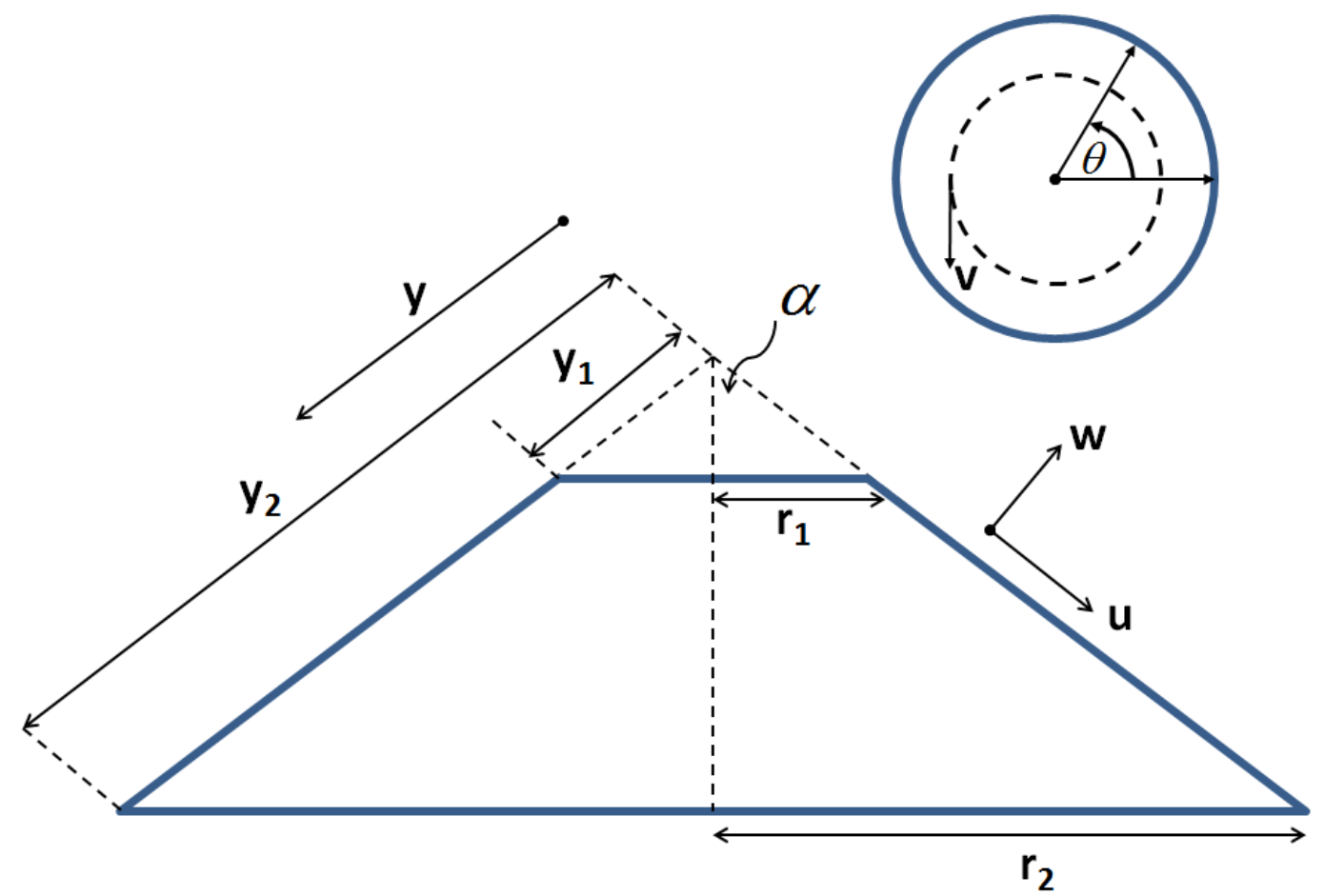

Figure A1: Coordinate system, dimensions, and displacement directions for the conical shell models.

\section{Matrix Coefficients}

The procedure for calculating the matrix coefficients in Eqs. 20, 28, 29, and 30 is detailed in this section. When Legendre polynomials are used as modal functions, matrix coefficients are given by:

$$
()=\frac{\left(y_{2}-y_{1}\right) \sin \alpha}{4} \int_{-1}^{1}()^{*} y d \eta
$$

where ()$^{*}$ are the coefficient integrands given below. Following from Eq. 16, the shell coordinate $y$ must be replaced in all integrations by the following:

$$
y=\frac{1}{2}\left(\left(y_{2}-y_{1}\right) \eta+\left(y_{2}+y_{1}\right)\right)
$$

For simply supported modal functions, the matrix coefficients are given by:

$$
()=\frac{\sin \alpha}{2} \int_{y_{1}}^{y_{2}}()^{*} y d y
$$

where ()$^{*}$ are the coefficient integrands given below, with $\frac{\partial \eta}{\partial y}=1$.

$$
\begin{aligned}
A^{*}(n, j ; k) & =\frac{2 E_{y} h \pi}{1-\nu_{y} \nu_{\theta}}\left(\frac{\partial \phi_{n}}{\partial \eta} \frac{\partial \phi_{i}}{\partial \eta}\left(\frac{\partial \eta}{\partial y}\right)^{2}+\frac{\nu_{\theta}}{2 y}\left(\frac{\partial \phi_{n}}{\partial \eta} \phi_{j} \frac{\partial \eta}{\partial y}+\frac{\partial \phi_{j}}{\partial \eta} \phi_{n} \frac{\partial \eta}{\partial y}\right)\right)+ \\
& \frac{2 E_{\theta} h \pi}{1-\nu_{y} \nu_{\theta}}\left(\frac{1}{y^{2}} \phi_{n} \phi_{j}+\frac{\nu_{y}}{2 y}\left(\frac{\partial \phi_{n}}{\partial \eta} \phi_{j} \frac{\partial \eta}{\partial y}+\frac{\partial \phi_{j}}{\partial \eta} \phi_{n} \frac{\partial \eta}{\partial y}\right)\right)+\frac{2 G_{y \theta} h k^{2} \pi}{y^{2} \sin ^{2} \alpha} \phi_{n} \phi_{j}
\end{aligned}
$$




$$
\begin{aligned}
& B^{*}(n, j ; k)=\frac{E_{y} h \pi \nu_{\theta} k}{1-\nu_{y} \nu_{\theta}}\left(\frac{1}{y \sin \alpha} \frac{\partial \phi_{n}}{\partial \eta} \phi_{j} \frac{\partial \eta}{\partial y}\right)+\frac{2 E_{\theta} h \pi k}{1-\nu_{y} \nu_{\theta}}\left(\frac{1}{y^{2} \sin \alpha} \phi_{n} \phi_{j}+\frac{\nu_{y}}{2 \mathrm{ysin} \alpha} \frac{\partial \phi_{n}}{\partial \eta} \phi_{j} \frac{\partial \eta}{\partial y}\right)+ \\
& 2 G_{y \theta} h \mathrm{k} \pi\left(\frac{1}{y^{2} \sin \alpha} \phi_{n} \phi_{j}-\frac{1}{\mathrm{y} \sin \alpha} \phi_{n} \frac{\partial \phi_{j}}{\partial \eta} \frac{\partial \eta}{\partial y}\right) \\
& C^{*}(n, j ; k)=\frac{2 E_{\theta} h \pi k^{2}}{1-\nu_{y} \nu_{\theta}}\left(\frac{1}{y^{2} \sin ^{2} \alpha} \phi_{n} \phi_{j}\right)+ \\
& 2 G_{y \theta} h \pi\left(\frac{\partial \phi_{n}}{\partial \eta} \frac{\partial \phi_{j}}{\partial \eta}\left(\frac{\partial \eta}{\partial y}\right)^{2}-\frac{1}{y}\left(\phi_{n} \frac{\partial \phi_{j}}{\partial \eta} \frac{\partial \eta}{\partial y}+\phi_{j} \frac{\partial \phi_{n}}{\partial \eta} \frac{\partial \eta}{\partial y}\right)+\frac{1}{y^{2}} \phi_{n} \phi_{j}\right) \\
& D^{*}(n, j ; k)=-\frac{E_{y} h \pi \nu_{\theta} \cot \alpha}{1-\nu_{y} \nu_{\theta}}\left(\frac{1}{y} \frac{\partial \phi_{n}}{\partial \eta} \phi_{j} \frac{\partial \eta}{\partial y}\right)-\frac{2 E_{\theta} h \pi \cot \alpha}{1-\nu_{y} \nu_{\theta}}\left(\frac{1}{y^{2}} \phi_{n} \phi_{j}+\frac{\nu_{y}}{2} \frac{1}{y} \frac{\partial \phi_{n}}{\partial \eta} \phi_{j} \frac{\partial \eta}{\partial y}\right) \\
& E(n, j ; k)=-\frac{2 E_{\theta} h \pi k \cot \alpha}{1-\nu_{y} \nu_{\theta}}\left(\frac{1}{y^{2} \sin \alpha} \phi_{n} \phi_{j}\right) \\
& F^{*}(n, j ; k)=\frac{2 E_{\theta} h \pi \cot ^{2} \alpha}{1-\nu_{y} \nu_{\theta}}\left(\frac{1}{y^{2}} \phi_{n} \phi_{j}\right)+2 D_{y} \pi\left(\frac{\partial^{2} \phi_{n}}{\partial \eta^{2}} \frac{\partial^{2} \phi_{j}}{\partial \eta^{2}}\left(\frac{\partial \eta}{\partial y}\right)^{4}\right)+ \\
& 2 D_{y} \pi\left\{\frac{\nu_{\theta}}{2 y}\left(\frac{\partial \phi_{n}}{\partial \eta} \frac{\partial^{2} \phi_{j}}{\partial \eta^{2}}\left(\frac{\partial \eta}{\partial y}\right)^{3}+\frac{\partial \phi_{j}}{\partial \eta} \frac{\partial^{2} \phi_{n}}{\partial \eta^{2}}\left(\frac{\partial \eta}{\partial y}\right)^{3}\right)-\frac{\nu_{\theta} k^{2}}{2 y^{2} \sin ^{2} \alpha}\left(\phi_{n} \frac{\partial^{2} \phi_{j}}{\partial \eta^{2}}\left(\frac{\partial \eta}{\partial y}\right)^{2}+\phi_{j} \frac{\partial^{2} \phi_{n}}{\partial \eta^{2}}\left(\frac{\partial \eta}{\partial y}\right)^{2}\right)\right\}+ \\
& 2 D_{\theta} \pi\left\{\frac{1}{y^{2}} \frac{\partial \phi_{n}}{\partial \eta} \frac{\partial \phi_{j}}{\partial \eta}\left(\frac{\partial \eta}{\partial y}\right)^{2}-\frac{k^{2}}{y^{3} \sin ^{2} \alpha}\left(\phi_{n} \frac{\partial \phi_{j}}{\partial \eta} \frac{\partial \eta}{\partial y}+\phi_{j} \frac{\partial \phi_{n}}{\partial \eta} \frac{\partial \eta}{\partial y}\right)+\frac{k^{4}}{y^{4} \sin ^{4} \alpha} \phi_{n} \phi_{j}\right\}+ \\
& 2 D_{\theta} \pi\left\{\frac{\nu_{y}}{2 y}\left(\frac{\partial \phi_{n}}{\partial \eta} \frac{\partial^{2} \phi_{j}}{\partial \eta^{2}}\left(\frac{\partial \eta}{\partial y}\right)^{3}+\frac{\partial \phi_{j}}{\partial \eta} \frac{\partial^{2} \phi_{n}}{\partial \eta^{2}}\left(\frac{\partial \eta}{\partial y}\right)^{3}\right)-\frac{\nu_{y} k^{2}}{2 y^{2} \sin ^{2} \alpha}\left(\phi_{n} \frac{\partial^{2} \phi_{j}}{\partial \eta^{2}}\left(\frac{\partial \eta}{\partial y}\right)^{2}+\phi_{j} \frac{\partial^{2} \phi_{n}}{\partial \eta^{2}}\left(\frac{\partial \eta}{\partial y}\right)^{2}\right)\right\}+ \\
& 4 D_{y \theta} \pi k^{2}\left\{\frac{1}{y^{2} \sin ^{2} \alpha} \frac{\partial \phi_{n}}{\partial \eta} \frac{\partial \phi_{j}}{\partial \eta}\left(\frac{\partial \eta}{\partial y}\right)^{2}-\frac{1}{y^{3} \sin ^{2} \alpha}\left(\phi_{n} \frac{\partial \phi_{j}}{\partial \eta} \frac{\partial \eta}{\partial y}+\phi_{j} \frac{\partial \phi_{n}}{\partial \eta} \frac{\partial \eta}{\partial y}\right)+\frac{1}{y^{4} \sin ^{2} \alpha} \phi_{n} \phi_{j}\right\} \\
& G^{*}(n, j)=2 m \pi \phi_{n} \phi_{j} \\
& H^{*}(n, j)=2 m \pi \phi_{n} \phi_{j} \\
& M^{*}(n, j)=2 m \pi \phi_{n} \phi_{j} \\
& J^{*}(n, j ; k)=-2 \tan \alpha p_{s} k^{2} \pi\left(\frac{3}{8 y \sin ^{2} \alpha} \phi_{n} \phi_{j}\right)-2 N_{y}^{a} k^{2} \pi\left(\frac{1}{4 y^{2} \sin ^{2} \alpha} \phi_{n} \phi_{j}\right) \\
& K^{*}(n, j ; k)=-\tan \alpha \mathrm{p}_{\mathrm{s}} \mathrm{k} \pi\left(\frac{3}{4 \sin \alpha} \phi_{n} \frac{\partial \phi_{j}}{\partial \eta} \frac{\partial \eta}{\partial y}+\frac{3}{4 \mathrm{ysin} \alpha} \phi_{n} \phi_{j}\right)-\mathrm{N}_{y}^{a} \mathrm{k} \pi\left(\frac{1}{2 \mathrm{ysin} \alpha} \phi_{n} \frac{\partial \phi_{j}}{\partial \eta} \frac{\partial \eta}{\partial y}+\frac{1}{2 \mathrm{y}^{2} \sin \alpha} \phi_{n} \phi_{j}\right) \\
& L^{*}(n, j ; k)=-2 \tan \alpha p_{\mathrm{s}} \pi\left(\frac{3}{8 y} \phi_{n} \phi_{j}+\frac{3}{8}\left(\phi_{n} \frac{\partial \phi_{j}}{\partial \eta} \frac{\partial \eta}{\partial y}+\phi_{j} \frac{\partial \phi_{n}}{\partial \eta} \frac{\partial \eta}{\partial y}\right)+\frac{3 y}{8} \frac{\partial \phi_{n}}{\partial \eta} \frac{\partial \phi_{j}}{\partial \eta}\left(\frac{\partial \eta}{\partial y}\right)^{2}+\frac{1}{y \tan ^{2} \alpha} \phi_{n} \phi_{j}\right)- \\
& 2 N_{y}^{a} \pi\left(\frac{1}{4 y^{2}} \phi_{n} \phi_{j}+\frac{1}{4 y}\left(\phi_{n} \frac{\partial \phi_{j}}{\partial \eta} \frac{\partial \eta}{\partial y}+\phi_{j} \frac{\partial \phi_{n}}{\partial \eta} \frac{\partial \eta}{\partial y}\right)+\frac{1}{4} \frac{\partial \phi_{n}}{\partial \eta} \frac{\partial \phi_{j}}{\partial \eta}\left(\frac{\partial \eta}{\partial y}\right)^{2}\right)
\end{aligned}
$$




$$
\begin{gathered}
N^{*}(n, j ; k)=\frac{2 p_{\mathrm{s}} k \pi}{\mathrm{y} \sin \alpha} \phi_{n} \phi_{j} \\
Q^{*}(n, j)=\frac{4 q \pi}{M}\left(\phi_{n} \frac{\partial \phi_{j}}{\partial \eta} \frac{\partial \eta}{\partial y}-\frac{1}{M y \sin \alpha} \phi_{n} \phi_{j}\right) \\
R^{*}(n, j ; k)=-2 \tan \alpha p_{\mathrm{s}} \pi\left(\frac{y}{2} \frac{\partial \phi_{n}}{\partial \eta} \frac{\partial \phi_{j}}{\partial \eta}\left(\frac{\partial \eta}{\partial y}\right)^{2}+\frac{k^{2}}{y^{2} \sin ^{2} \alpha} \phi_{n} \phi_{j}\right)-2 N_{y}^{a} \pi \frac{\partial \phi_{n}}{\partial \eta} \frac{\partial \phi_{j}}{\partial \eta}\left(\frac{\partial \eta}{\partial y}\right)^{2} \\
S(n, j)=\left.\left.K_{s} \pi \sin \alpha \sum_{i} \mathrm{y}\right|_{y_{i}} \phi_{n} \phi_{j}\right|_{\eta_{i}}
\end{gathered}
$$

Note: $\mathrm{S}(\mathrm{n}, \mathrm{j})$ is not integrated.

$$
\begin{gathered}
V^{P Y R^{*}}(n, j)=2 \pi m^{P y r} \phi_{n} \phi_{j} \\
Y^{P Y R^{*}}(n, j)=2 \pi K^{P y r} \phi_{n} \phi_{j} \\
Z_{A}^{*}(n, j)=\frac{4 q \pi}{M U_{\infty}} \phi_{n} \phi_{j} \\
Z_{S D}(n, j)=2 \zeta_{n} \omega_{n} M(n, j)
\end{gathered}
$$

Note: $Z_{S D}$ is not integrated.

\section{References}

${ }^{1}$ Hughes, S. J., Cheatwood, F. M., Calomino, A. M., and Wright, H. S., "Hypersonic Inflatable Aerodynamic Decelerator (HIAD) Technology Development Overview," 2013.

${ }^{2}$ Hughes, S. J., Ware, J. S., Del Corso, J. A., and Lugo, R. A., "Deployable Aeroshell Flexible Thermal Protection System Testing," AIAA Aerodynamic Decelerator Systems Technology Conference and Seminar, AIAA-2009-2926, 2009.

${ }^{3}$ Del Corso, J. A., Cheatwood, F., Bruce, W., Hughes, S. J., and Calomino, A. M., "Advanced High-Temperature Flexible TPS for Inflatable Aerodynamic Decelerators," 21st AIAA Aerodynamic Decelerator Systems Technology Conference and Seminar, Vol. 1, 2011, pp. 139-161.

${ }^{4}$ Goldman, B. D., Scott, R. C., and Dowell, E. H., "Nonlinear Aeroelastic Analysis of the HIAD TPS Coupon in the NASA 8' High Temperature Tunnel: Theory and Experiment," 2013 (submitted).

${ }^{5}$ Goldman, B. D., Dowell, E. H., and Scott, R. C., "Flutter Analysis of the Thermal Protection Layer on the NASA HIAD," Proc., 22nd AIAA Aerodynamic Decelerator Systems (ADS) Conf., 2013.

${ }^{6}$ Shulman, Y., Vibration and Flutter of Cylindrical and Conical Shells, Air Force Office of Scientific Research, Air Research and Development Command, United States Air Force, 1959.

${ }^{7}$ Dixon, S. C. and Hudson, M. L., Flutter, Vibration, and Buckling of Truncated Orthotropic Conical Shells with Generalized Elastic Edge Restraint, National Aeronautics and Space Administration, 1970.

${ }^{8}$ Sabri, F. and Lakis, A. A., "Hybrid Finite Element Method Applied to Supersonic Flutter of an Empty or Partially Liquid-Filled Truncated Conical Shell," Journal of Sound and Vibration, Vol. 329, No. 3, 2010, pp. $302-316$.

${ }^{9}$ Bismarck-Nasr, M. N. and Costa Savio, H. R., "Finite-Element Solution of the Supersonic Flutter of Conical shells," AIAA Journal, Vol. 17, No. 10, 1979, pp. 1148-1150.

${ }^{10}$ Sunder, P., Ramakrishnan, C., and Sengupta, S., "Optimum Cone Angles in Aeroelastic Flutter," Computers Es Structures, Vol. 17, No. 1, 1983, pp. 25-29.

${ }^{11}$ NASA, "HIAD Image Gallery," http://www.nasa.gov/offices/oct/stp/game_changing_development/HIAD/images . html.

${ }^{12} 3 \mathrm{M}$, "Ceramic Textiles and Composites," Tech. rep., 3M.

${ }^{13}$ Fish, J., Yu, Q., and Shek, K., "Computational Damage Mechanics for Composite Materials Based on Mathematical Homogenization," International journal for numerical methods in engineering, Vol. 45, No. 11, 1999, pp. $1657-1679$.

${ }^{14}$ Dupont, "Kevlar Technical Guide," Tech. rep., Dupont.

${ }^{15}$ Matweb, "DuPont Kevlar 49 Aramid Fiber," July 2013.

${ }^{16}$ Matweb, "MarkeTech Silica Aerogel," July 2013. 
${ }^{17}$ Seide, P., "A Donnell-Type Theory for Asymmetrical Bending and Buckling of Thin Conical Shells," Journal of Applied Mechanics, Vol. 24, No. 4, 1957, pp. 547-552.

${ }^{18}$ MacNeal, R. H., The NASTRAN Theoretical Manual, Vol. 1, Scientific and Technical Information Office, National Aeronautics and Space Administration, 1972.

${ }^{19}$ Krumhaar, H., "The Accuracy of Linear Piston Theory When Applied to Cylindrical Shells," AIAA Journal, Vol. 1, No. 6, 1963, pp. 1448-1449.

${ }^{20} \mathrm{Hu}$, W., Gormley, J., and Lindholm, U., "Flexural Vibrations of Conical Shells with Free Edges." Tech. rep., DTIC Document, 1966.

${ }^{21}$ Dowell, E. H. and Bliss, D. B., "New Look at Unsteady Supersonic Potential Flow Aerodynamics and Piston Theory," AIAA Journal, Vol. 51, No. 9, 2013, pp. 2278-2281. 